\title{
RIGOROUS COMPUTATION OF THE GLOBAL DYNAMICS OF INTEGRODIFFERENCE EQUATIONS WITH SMOOTH NONLINEARITIES*
}

\author{
SARAH DAY ${ }^{\dagger}$ AND WILLIAM D. KALIES ${ }^{\ddagger}$
}

\begin{abstract}
Topological tools, such as Conley index theory, have inspired rigorous computational methods for studying dynamics. These methods rely on the construction of an outer approximation, a combinatorial representation of the system that incorporates small, bounded error. In this work, we present an automated approach to constructing outer approximations for systems in a class of integrodifference operators with smooth nonlinearities. Chebyshev interpolants and Galerkin projections form the basis for the construction, while analysis and interval arithmetic are used to incorporate explicit error bounds. This represents a significant advance to the approach given by Day, Junge, and Mischaikow [SIAM J. Appl. Dyn. Syst., 3 (2004), pp. 117-160], extending the nonlinearities that may be studied from low degree polynomials to smooth functions and the studied portion of phase space from a simulated attracting region to the global maximal invariant set. As a demonstration of the techniques, a Morse decomposition of the global dynamics, a list of validated periodic orbits, semiconjugate symbolic dynamics, and a lower bound on topological entropy are computed for the Kot-Schaffer integrodifference operator from ecology with the exponential Ricker nonlinearity.
\end{abstract}

Key words. outer approximation, global dynamics, Conley index

AMS subject classifications. 37L65, 37M25, 37B30, 37N25

DOI. $10.1137 / 120903129$

1. Introduction. The aim of this paper is to present a computational scheme to rigorously bound and analyze global dynamics for systems from a broad class of discrete-time, growth-dispersal models given as integrodifference equations. More explicitly, we consider a map $\Phi$ on $L^{2}([-\pi, \pi])$, of the form

$$
\Phi[a](y):=\frac{1}{2 \pi} \int_{-\pi}^{\pi} b(x, y) G[a](x) d x
$$

where $G$ is an $L^{\infty}$-bounded operator that is well approximated by a polynomial Nemytskii operator with computable error bounds (as described later in this section). We also require $b(x, y)=b(x-y)$ to be smooth. In this case, the maximal invariant set under $\Phi$ is compact, and we construct a subset of Fourier space on which we use analytic estimates to compute and refine bounds on the maximal invariant set in order to study global dynamics. A coarse, but rigorous, combinatorial representation of the dynamics is given by an outer approximation, a multivalued representation of the studied system at a fixed resolution with incorporated bounded error. Essentially, the outer approximation encodes outer bounds on images of sets under (1.1). Topological tools such as Conley index theory may then be applied to draw rigorous conclusions about the dynamics. Examples of dynamics attainable using outer approximation and Conley index theory include Morse decompositions, inner

*Received by the editors December 19, 2012; accepted for publication (in revised form) July 29, 2013; published electronically November 7, 2013.

http://www.siam.org/journals/sinum/51-6/90312.html

${ }^{\dagger}$ Department of Mathematics, College of William and Mary, Williamsburg, VA 23187 (sday@ math.wm.edu). This author was partially supported by NSF grants DMS-0955604 and DMS-0811370.

${ }^{\ddagger}$ Department of Mathematical Sciences, Florida Atlantic University, Boca Raton, FL 33431 (wkalies@fau.edu). This author was partially supported by NSF grant DMS-0914995. 
approximation of basins of attraction, and the existence of invariant sets of varying stability types and ranging from fixed points to chaotic dynamics. For more information on using Conley index theory and outer approximations, see $[13,6,7,5,11,1,2]$ and references therein.

To illustrate the techniques presented in this paper, we also present sample computations and results for the Kot-Schaffer map with Ricker nonlinearity. Kot and Schaffer proposed (1.1) as a general model for populations with synchronous, nonoverlapping generations and distinct phases for (relatively sedentary) growth and (nonsedentary) dispersal [12]. For our example, $b$ is a dispersal kernel, and $G$ is the nonlinear Ricker growth operator

$$
G[a](x)=\mu c(x) a(x) e^{-a(x)},
$$

where $\mu$ is a bifurcation (fitness) parameter and $c \in C^{2}([-\pi, \pi])$, a nonnegative function with $\|c\|_{\infty}=1$, models heterogeneous variation in the fitness of the environment. Since this is a population model, the natural phase space consists of the nonnegative functions. In order to use topological methods to study the dynamics on this space, including its boundary, we choose a fixed, small $\epsilon>0$ and set the domain to be $L^{2}([-\pi, \pi],(-\epsilon, \infty))$. We use this model to demonstrate the computational results that can be obtained, but the methods apply in the more general setting of (1.1).

In [6], Day, Junge, and Mischaikow obtained sample results on the attractor for the Kot-Schaffer map with logistic nonlinearity $G[a]=\mu a\left(1-\frac{a}{c}\right)$, where $\mu>0$ and $c \in L^{2}[-\pi, \pi]$. The key differences between that work and this project are twofold: first, the approach has been extended to allow for a more systematic study of the global invariant set rather than individual (smaller) invariant sets, and second, we incorporate a polynomial approximation of $G$ to allow for the study here of a much broader class of nonlinearities, including the exponential Ricker operator. The extension of the study to the global maximal invariant set is obtained by the incorporation of regularity estimates and an automated, adaptive approach to refining bounds on this set. The extension to smooth nonlinearities follows from the incorporation of Chebyshev interpolants to approximate the nonlinearity.

In the following sections we develop a theoretical framework in which we perform the calculations and provide computable error bounds. To make the estimates more concise, we consider the special case in which $G$ is a Nemystkii operator of the form $G[a](x)=c(x) g(a(x))$ for some smooth $c:[-\pi, \pi] \rightarrow \mathbb{R}$ and $g: \mathbb{R} \rightarrow \mathbb{R}$ that is bounded and smooth. Chebyshev interpolation is used to obtain a polynomial approximation of $G$ with explicit error bounds. The resulting framework developed here has a natural extension to the more general setting where $G$ can be approximated by a polynomial Nemytskii operator of the form $p(x, a(x))=c_{0}(x)+c_{1}(x) a(x)+\cdots+c_{N}(x) a^{N}(x)$ with an explicitly computable error bound. Even though these techniques apply to systems in this broad class, for clarity we do not present the estimates in this generality. For ease of presentation we also restrict our attention to the space of even functions. However, all of the results also hold without this restriction. We also implement these ideas for the map (1.2) and demonstrate that the general procedure is automated and, beyond some preliminary work, independent of the particular operator chosen for study from this class.

This approach to constructing a feasible computational framework for the system has three key steps. The first is to use regularity arguments to restrict the domain to a compact region of phase space. The second step is to approximate the nonlinearity $G$ using Chebyshev interpolants and to bound the error in performing this 
approximation. The third step is to decompose the map in a Fourier basis, using Galerkin projection to establish a finite-dimensional system for numerical work and computing truncation error bounds in both the finite-dimensional system and the remaining modes. While the first two steps are independent of one another, the third follows and builds on the first two. The resulting constructed system is well suited to analysis by topological tools such as Conley index theory. In what follows, we use analysis based on regularity and related arguments to construct bounds on the global maximal invariant set in section 2, present necessary background from Conley index theory in section 3, give the framework based on Chebyshev interpolants and Galerkin projections and their associated error bounds for the approximation of $\Phi$ in sections 4 through 6 , and finally combine these pieces into one cohesive computational approach in section 7 . We conclude with sample computations in section 8 . Our primary goal here is to provide explicit computational bounds (avoiding longer, more technical, calculations) as we demonstrate the feasibility of the approach. Many of the inequalities, especially those for the bounds of Galerkin truncation errors, could be improved to provide tighter bounds if needed; cf. Appendix A in [19].

2. Global bounds on the dynamics. To capture the entire global dynamics, we need to establish reasonable, computable a priori bounds on the maximal invariant set for the system. In this approach, we compute a compact cover of the maximal invariant set and use this portion of phase space for a more detailed computational study.

We will now establish two sets of bounds. The first uses regularity properties of the dispersal kernel to compute bounds on the Fourier coefficients of functions in the maximal invariant set. Bounds of this type will exhibit power decay. The second set of bounds exploits the form of the nonlinearity to find additional pointwise bounds on functions in the maximal invariant set. Throughout, we illustrate the estimates with the Kot-Schaffer map with Ricker nonlinearity as an example.

2.1. Regularity bounds. We now derive a priori bounds on the Fourier coefficients for any function $z \in L^{2}([-\pi, \pi])$ which is in the image of $\Phi$. Hence these bounds apply to all functions in the maximal invariant set. For $a \in L^{2}([-\pi, \pi])$, let

$$
z(y)=\Phi[a](y)=\frac{1}{2 \pi} \int_{-\pi}^{\pi} b(x, y) G[a](x) d x .
$$

Then for an integer $s \geq 0$

$$
z^{(s)}(y)=\frac{1}{2 \pi} \int_{-\pi}^{\pi}\left[\frac{\partial^{s}}{\partial y^{s}} b(x, y)\right] G[a](x) d x .
$$

Set

$$
B_{\max }^{(s)} \geq \max _{(x, y) \in[-\pi, \pi]^{2}}\left|\frac{\partial^{s}}{\partial y^{s}} b(x, y)\right|
$$

and

$$
G_{\max } \geq \sup _{a \in \Omega}\|G[a]\|_{L^{\infty}}
$$

Then

$$
\left\|z^{(s)}\right\|_{L^{1}}=\int_{-\pi}^{\pi}\left|z^{(s)}(y)\right| d y=\frac{1}{2 \pi} \int_{-\pi}^{\pi}\left|\int_{-\pi}^{\pi}\left[\frac{\partial^{s}}{\partial y^{s}} b(x, y)\right] G[a](x) d x\right| d y
$$




$$
\begin{aligned}
& \leq \frac{1}{2 \pi} \int_{-\pi}^{\pi} \int_{-\pi}^{\pi} B_{\max }^{(s)} G_{\max } d x d y \\
& \leq 2 \pi B_{\max }^{(s)} G_{\max } .
\end{aligned}
$$

Furthermore,

$$
\left[z^{(s)}(y)\right]^{2}=\frac{1}{2 \pi} \int_{-\pi}^{\pi}\left[\frac{\partial^{s}}{\partial y^{s}} b(x, y)\right] z^{(s)}(y) G[a](x) d x
$$

so that

$$
\begin{aligned}
\left\|z^{(s)}\right\|_{L^{2}}^{2}=\int_{-\pi}^{\pi}\left|z^{(s)}(y)\right|^{2} d y & =\frac{1}{2 \pi} \int_{-\pi}^{\pi}\left|\int_{-\pi}^{\pi}\left[\frac{\partial^{s}}{\partial y^{s}} b(x, y)\right] G[a](x) d x z^{(s)}(y)\right| d y \\
& \leq \frac{G_{\max }}{2 \pi} \int_{-\pi}^{\pi} \int_{-\pi}^{\pi}\left|\frac{\partial^{s}}{\partial y^{s}} b(x, y)\right|\left|z^{(s)}(y)\right| d x d y \\
& \leq \frac{G_{\max } B_{\max }^{(s)}}{2 \pi} \cdot 2 \pi\left\|z^{(s)}\right\|_{L^{1}} \\
& \leq 2 \pi\left(B_{\max }^{(s)} G_{\max }\right)^{2} .
\end{aligned}
$$

Therefore, for any function $z \in \Phi\left(L^{2}([-\pi, \pi])\right)$ we have $\left\|z^{(s)}\right\|_{L^{2}} \leq \sqrt{2 \pi} B_{\max }^{(s)} G_{\max }$. Now let $a(x)=\sum_{k} a_{k} e^{i k x}$ and $z(y)=\sum_{k} z_{k} e^{i k y}$ be the Fourier expansions for $a$ and $z=\Phi[a]$, respectively. Then for each $n \in \mathbb{Z}$

$$
\begin{aligned}
|n|^{2 s}\left|z_{n}\right|^{2} & \leq \sum_{k}\left|(i k)^{s} z_{k}\right|^{2}=\frac{1}{2 \pi}\left\|z^{(s)}\right\|_{L^{2}}^{2} \\
& \leq \frac{1}{2 \pi}\left(\sqrt{2 \pi} B_{\max }^{(s)} G_{\max }\right)^{2} \\
& \leq\left(B_{\max }^{(s)} G_{\max }\right)^{2}
\end{aligned}
$$

and hence

$$
\left|z_{n}\right| \leq \begin{cases}\frac{B_{\max }^{(s)} G_{\max }}{|n|^{s}}, & n \neq 0 \\ B_{\max }^{(0)} G_{\max }, & n=0\end{cases}
$$

Thus, the maximal invariant set $S$ of $\Phi$ satisfies for each $a \in S$

$$
\left|a_{n}\right| \leq\left\{\begin{array}{cc}
\frac{A_{s}}{|n|^{s}}, & n \neq 0 \\
A_{s}, & n=0
\end{array}\right.
$$

where $s \geq 0$ and $A_{s}=\max \left\{B_{\max }^{(s)} G_{\max }, B_{\max }^{(0)} G_{\max }\right\}$.

Example. For the special case of the Ricker nonlinearity where $G[a](x)=\mu c(x)$ $a(x) e^{-a(x)}$, we may take $G_{\max }:=\mu / e$ since $\max _{x \in[-\pi, \pi]}|c(x)|=1$ by assumption. The bounds $B_{\max }^{(s)}$ are obtained in the next section. 
2.2. Explicit kernel bounds. We now compute bounds $B_{\max }^{(s)}$ for a specific class of dispersal kernels. Suppose

$$
b(x, y)=b(x-y)=\sum_{k} b_{k} e^{i k(x-y)} .
$$

Then

$$
\frac{\partial^{s}}{\partial y^{s}} b(x, y)=\sum_{k}(-i k)^{s} b_{k} e^{i k(x-y)}
$$

Thus, we may set

$$
B_{\max }^{(s)} \geq \sum_{k}|k|^{s}\left|b_{k}\right| \cdot
$$

We assume an exponential decay of the Fourier modes for the dispersal kernel so that there exist $\lambda>1$ and $K>0$ such that $\left|b_{k}\right| \leq \lambda^{-|k|}$ for all $k \geq K$. Then for a fixed computational parameter $N>K$ we set

$$
\begin{aligned}
B_{\max }^{(s)}=B_{\max }^{(s)}(N) & :=2 \sum_{k=1}^{N} k^{s}\left|b_{k}\right|+\frac{1}{\lambda^{N-1}} \sum_{j=0}^{s} \frac{s ! N^{s-j}}{(s-j) !(\ln \lambda)^{j+1}} \\
& =2 \sum_{k=1}^{N} k^{s}\left|b_{k}\right|+2 \int_{N}^{\infty} \frac{x^{s}}{\lambda^{x}} d x \\
& \geq \sum_{k}|k|^{s}\left|b_{k}\right|,
\end{aligned}
$$

except for the case $s=0$, where the tail bound can be summed exactly.

Example. In section 8 we compute on the Kot-Schaffer map with Ricker nonlinearity. For the dispersal kernel in those computations we use $\lambda=15, \mu=30$, and $s=10$ to bound modes $n \geq 30$. Using $N=200$ for these computations yields $B_{\max }^{(10)}=46.8$ and $A_{10}=1403.99$, providing a global bound of

$$
\left|a_{n}\right| \leq \frac{1403.99}{|n|^{10}}=\frac{A_{10}}{|n|^{10}} .
$$

The above bound on $\left|a_{30}\right|$ is approximately $2.4 \times 10^{-12}$. This is not the minimal bound for $n=30$. As $n$ increases, the minimal estimate is obtained by increasing $s$. The minimal bound for $n=30$ is $\left|a_{30}\right| \leq 2.6 \times 10^{-32}$ using $s=53$, as shown in Figure 2.1.

2.3. Pointwise bounds. We now define pointwise bounds on $G[a](x)$ which will be used to further refine the bounds on the maximal invariant set. Let

$$
G_{\min }(x) \leq \inf _{a \in L^{2}([-\pi, \pi])} G[a](x) \quad \text { and } \quad G_{\max }(x) \geq \sup _{a \in L^{2}([-\pi, \pi])} G[a](x) .
$$

Then for $b(x, y) \geq 0$ we have the following bounding functions:

$$
\frac{1}{2 \pi} \int_{-\pi}^{\pi} b(x, y) G_{\min }(x) d x \leq \Phi[a](y) \leq \frac{1}{2 \pi} \int_{-\pi}^{\pi} b(x, y) G_{\max }(x) d x .
$$

The above inequality gives pointwise bounds on any function $z=\Phi[a]$ in the global invariant set. If the kernel $b$ changes sign, then cruder bounds obtained by replacing $b(x, y)$ by $\pm|b(x, y)|$ may be used.

Example. For the computations in section 8, given the domain $L^{2}([-\pi, \pi],(-\epsilon, \infty))$ and Ricker nonlinearity $G[a](x)=\mu c(x) a(x) e^{-a(x)}$, we take $G_{\min }(x):=-\mu \epsilon e^{\epsilon}$ and $G_{\max }(x)=\mu c(x) / e$. 




FIG. 2.1. Curves $A_{s} / n^{s}$ for $s=1, \ldots, 55$ on a semilog scale. Minimum envelope is in bold.

2.4. Global bounds in practice. We now combine the bounds presented in sections 2.1 and 2.3 in order to construct and refine a compact subset of the phase space on which computations will take place. By section 2.1, we may choose a power decay rate, $s \geq 2$, and an associated constant, $A_{s} \geq 0$, such that the Fourier expansion $a(x)=\sum_{n} a_{n} e^{i n x}$ of every function in the maximal invariant set satisfies

$$
a_{n} \in \begin{cases}A_{s}[-1,1], & n=0 \\ \frac{A_{s}}{|n|^{s}}[-1,1], & n \neq 0\end{cases}
$$

In practice, we choose a computational dimension $M$ and designate the Fourier modes $0, \ldots, M-1$ as computational modes and the remaining modes as tail modes. Then we may use multiple $\left(s, A_{s}\right)$ pairs to define a compact bounding region for the maximal invariant set of the type

$$
Z=\frac{A_{s_{0}}}{n^{s_{0}}}[-1,1] \times \cdots \times \frac{A_{s_{M-1}}}{n^{s_{M-1}}}[-1,1] \times \prod_{n \geq M} \frac{A_{s_{*}}}{n^{s_{*}}}[-1,1]
$$

by minimizing the bound in (2.1); see Figure 2.1. Thus, $s_{*}$ is the largest $s$ such that the estimate in (2.1) holds for all $n \geq M$. The regularity estimates imply that the subspace of $L^{2}(-\pi, \pi)$ corresponding to $Z$ is forward invariant under $\Phi$, and hence computational work may now be restricted to this region. For ease of presentation we have restricted our attention to the space of even functions for which $a_{-n}=a_{n}$. However, all of the results also hold without this restriction.

The results of section 2.3 allow us to further refine this set in the computational modes. For $a$ in the maximal invariant set and $y \in[-\pi, \pi]$, we have that

$$
A_{\min }(y) \leq a(y) \leq A_{\max }(y),
$$

where $A_{\min / \max }(y):=\frac{1}{2 \pi} \int_{-\pi}^{\pi} b(x, y) G_{\min / \max }(x) d x$ as in (2.3). In practice, we choose $y_{1}, y_{2}, \ldots, y_{k} \in[-\pi, \pi]$ and consider bounds on $a\left(y_{j}\right)$ for functions $a$ whose Fourier coefficients lie in an infinite-dimensional box of the form

$$
B=\prod_{0 \leq n<M} \tilde{a}_{n} \times \prod_{n \geq M} \frac{A_{s_{*}}}{n^{s_{*}}}[-1,1] \subseteq Z .
$$

In general, we will use a tilde above a variable to denote an interval. In this case $\tilde{a}_{n}$ is a subinterval of $\frac{A_{s_{n}}}{|n|^{s_{n}}}[-1,1]$, and hence $\prod \tilde{a}_{n}$ is a rectangular parallelepiped in $\mathbb{R}^{M}$. 
For a function $a$ with Fourier coefficients in $B$ and using $a_{-n}=a_{n}$, we have

$$
\begin{aligned}
a\left(y_{j}\right) & \in \sum_{n<M} 2 \tilde{a}_{n} \cos \left(n y_{j}\right)+\sum_{|n| \geq M} \frac{A_{s_{*}} e^{i n y_{j}}}{|n|^{s_{*}}}[-1,1] \\
& \subset \sum_{n<M} 2 \tilde{a}_{n} \cos \left(n y_{j}\right)+2 \int_{M-1}^{\infty} \frac{A_{s_{*}}}{x^{s_{*}}} d x[-1,1] \\
& =\sum_{n<M} 2 \tilde{a}_{n} \cos \left(n y_{j}\right)+\frac{2 A_{s_{*}}}{\left(s_{*}-1\right)(M-1)^{s_{*}-1}}[-1,1] .
\end{aligned}
$$

If

$$
\left(\sum_{n<M} 2 \tilde{a}_{n} \cos \left(n y_{j}\right)+\frac{2 A_{s_{*}}}{\left(s_{*}-1\right)(M-1)^{s_{*}-1}}[-1,1]\right) \cap\left[A_{\min }\left(y_{j}\right), A_{\max }\left(y_{j}\right)\right]=\emptyset
$$

then $B$ does not intersect the set of functions satisfying the inequality (2.3) and will not contain any functions in the maximal invariant set of $\Phi$. Therefore, we may remove $B$ from a covering of the maximal invariant set. These bounds can dramatically decrease the size of the set on which computations need to be performed; see sections 7 and 8 .

3. Outer approximations and computational Conley index theory. An outer approximation is a finite representation of a dynamical system that is compatible with tools from Conley index theory in two ways. First, it incorporates round-off and other bounded errors that occur in its construction so that the derived results are rigorous. Second, it is a combinatorial object to which tools from computational topology and graph theory can be applied. In this section, we briefly discuss outer approximations and the use of Conley index theory to uncover dynamics. Since the focus of this work is on new techniques for constructing outer approximations for infinite-dimensional maps, we keep the discussion of Conley index theory brief and refer the reader to $[11,6,5,1]$ and references therein for more details.

We begin the construction of an outer approximation by decomposing the phase space into a finite (combinatorial) list of objects on which we compute image bounds. Following the approach in section 2, if $Z$ is the domain in Fourier space on which we perform the computations, there is a topological conjugacy between the map $\Phi$ restricted to the inverse Fourier transform of $Z$ as a subset of $\Omega$ and the map on $Z$ expressed in Fourier space. Therefore, we study the map in Fourier space, which is described in detail in section 4. Equation (2.4) in section 2.4 provides a compact bounding set for the maximal invariant set expressed in Fourier modes. Also combining (2.4) with the splitting into computational and tail modes, we have that the maximal invariant set is contained in a set of the form $Z=R \times V$, where $R=\prod_{0 \leq n<M}\left[a_{n}^{-}, a_{n}^{+}\right] \subset \mathbb{R}^{M}$ is a rectangular bounding box for the computational modes, and for some $s_{*} \geq 2$ and $A_{s_{*}}>0, V=\prod_{n \geq M} \frac{A_{s_{*}}}{n^{s_{*}}}[-1,1]$ is a bounding set for the tail modes.

Our primary method for decomposing the phase space is subdivision of the bounding box in the computational modes. This yields a cubical grid

$$
\mathcal{Z}:=\left\{\prod_{n=0}^{M-1}\left[a_{n}^{-}+\frac{i_{n} r_{n}}{2^{d_{n}+1}}, a_{n}^{-}+\frac{\left(i_{n}+1\right) r_{n}}{2^{d_{n}+1}}\right] \times V \mid i_{n} \in\left\{0, \ldots, 2^{d_{n}}-1\right\}\right\},
$$


where $r_{n}=\left(a_{n}^{+}-a_{n}^{-}\right) / 2$ is the radius of $R$ in the $n$th coordinate and the number of subdivisions in mode $n, d_{n}$, is a nonnegative integer. In practice, we choose to subdivide some of the computational modes more than others based on error bound calculations (see section 7 for more details). We refer to the set represented by an element of $\mathcal{Z}, B=\prod_{n=0}^{M-1}\left[a_{n}^{-}+\frac{i_{n} r_{n}}{2^{d_{n}}}, a_{n}^{-}+\frac{\left(i_{n}+1\right) r_{n}}{2^{d_{n}}}\right] \times V$, as a box. For a collection of boxes, $\mathcal{B} \subset \mathcal{Z}$, define the topological realization of $\mathcal{B}$ as $|\mathcal{B}|:=\cup_{B \in \mathcal{B}} B \subset \mathbb{R}^{M} \times V$.

Constructing an outer approximation on $\mathcal{Z}$ involves computing an outer bound on the image $\Phi(B)$ for each $B \in \mathcal{Z}$. In our approach, we use a combination of analytic error bounds and interval arithmetic calculations to compute an outer bound on $\Phi(B)$ which we then intersect with the grid $\mathcal{X}$. The corresponding outer approximation is a multivalued map $\mathcal{F}: \mathcal{Z} \rightrightarrows \mathcal{Z}$, where for $B \in \mathcal{Z}$

$$
\mathcal{F}(B) \supset\left\{B^{\prime} \in \mathcal{X} \mid B^{\prime} \cap \Phi(B) \neq \emptyset\right\} .
$$

The outer approximation $\mathcal{F}$ can be represented by a directed graph for computational purposes. In the directed graph representation, the vertex set is the collection of boxes in the grid and there is a directed edge from vertex $B$ to vertex $B^{\prime}$ if and only if $B^{\prime} \in \mathcal{F}(B)$. Any continuous function satisfying

$$
f(x) \in \mathcal{F}(B) \quad \text { for any } x, B \in \mathcal{Z} \text { with } x \in B
$$

is a continuous selector for $\mathcal{F}$. By construction, $\Phi$ (in the Fourier basis) is a continuous selector for $\mathcal{F}$.

3.1. Computational Conley index theory. Computational Conley index theory is one tool that can be applied to outer approximations in order to draw rigorous conclusions about the dynamics of the original system. We now briefly discuss this tool, beginning with an extension of dynamical systems terminology to outer approximations followed by the definitions of isolating neighborhood and index pair, the building blocks of Conley index theory.

Definition 3.1. A combinatorial trajectory of a multivalued map $\mathcal{F}$ through $B \in \mathcal{Z}$ is a bi-infinite sequence $\gamma_{B}=\left(\ldots, B_{-1}, B_{0}, B_{1}, \ldots\right)$ with $B_{0}=B, B_{n} \in \mathcal{Z}$, and $B_{n+1} \in \mathcal{F}\left(B_{n}\right)$ for all $n \in \mathbb{Z}$.

Let $f: X \rightarrow X$ be a continuous map. A trajectory through $x \in X$ is a sequence

$$
\gamma_{x}:=\left(\ldots, x_{-1}, x_{0}, x_{1}, \ldots\right)
$$

such that $x_{0}=x$ and $x_{n+1}=f\left(x_{n}\right)$ for all $n \in \mathbb{Z}$. Note that given an outer approximation $\mathcal{F}$ of $f$ and a trajectory $\gamma_{x}:=\left(\ldots, x_{-1}, x_{0}, x_{1}, \ldots\right)$ of $f, \gamma_{B}=\left(\ldots, B_{-1}, B_{0}\right.$, $\left.B_{1}, \ldots\right)$, where $x_{i} \in B_{i}$ is a trajectory for $\mathcal{F}$. We now define the invariant set relative to $N \subset X$ as

$$
\operatorname{Inv}(N, f):=\left\{x \in N \mid \text { there exists a trajectory } \gamma_{x} \text { with } \gamma_{x} \subset N\right\} .
$$

Definition 3.2. The combinatorial invariant set in $\mathcal{N} \subset \mathcal{Z}$ for a multivalued map $\mathcal{F}$ is

$$
\operatorname{Inv}(\mathcal{N}, \mathcal{F}):=\left\{B \in \mathcal{Z} \mid \text { there exists a trajectory } \gamma_{B} \subset \mathcal{N}\right\} .
$$

Conley index theory measures invariant sets in isolating neighborhoods.

Definition 3.3. Let $X$ be a locally compact metric space. A compact set $N \subset X$ is an isolating neighborhood for $g: X \rightarrow X$ if

$$
\operatorname{Inv}(N, g) \subset \operatorname{int}(N),
$$


where $\operatorname{int}(N)$ denotes the interior of $N$. A set $S$ is an isolated invariant set if $S=$ $\operatorname{Inv}(N, f)$ for some isolating neighborhood $N$.

In our approach, we construct compact sets of the form $N=N_{F} \times V \subset \mathbb{R}^{M} \times V$. By Theorem 2.3 in [6], if

1. $N_{F}$ is an isolating neighborhood for any $g: \mathbb{R}^{M} \rightarrow \mathbb{R}^{M}$ such that for all $x \in N_{F}, g(x) \in \pi(f(x \times V))$, where $\pi: \mathbb{R}^{M} \times V \rightarrow \mathbb{R}^{M}$ is the projection map, and

2. $V$ is forward invariant under $f$, that is, $\bar{\pi}(f(N)) \subset V$, where $\bar{\pi}: N_{F} \times V \rightarrow V$ is projection onto $V$,

then $N$ is an isolating neighborhood for $f: \mathbb{R}^{M} \times V \rightarrow \mathbb{R}^{M} \times V$.

While there are different sufficient conditions for isolation in the setting of outer approximations, we chose the following for this work. The set $o(\mathcal{B}):=\{B \in \mathcal{Z} \mid B \cap$ $|\mathcal{B}| \neq \emptyset\}$, sometimes referred to as a one box neighborhood of $\mathcal{B}$ in $\mathcal{Z}$, provides the smallest representable neighborhood $|o(\mathcal{B})|$ of $|\mathcal{B}|$ in the grid $\mathcal{Z}$. If

$$
o(\operatorname{Inv}(\mathcal{N}, \mathcal{F})) \subset \mathcal{N}
$$

then $\mathcal{N} \subset \mathcal{Z}$ is a combinatorial isolating neighborhood under $\mathcal{F}$. Note that this combines the notion of computable isolation in the refined computational modes with the construction of the grid $\mathcal{Z}$ on a global bound set for the maximal invariant set. That is, forward invariance of continuous selectors on $|\mathcal{Z}|$ is built into the outer approximation, and this is upheld by the continuous selector we wish to study, $\Phi$. For example, $o(\mathcal{Z})=\mathcal{Z}$ and $\mathcal{Z}$ is a combinatorial isolating neighborhood for $\mathcal{F}$.

By construction, the topological realization $|\mathcal{N}|=N_{F} \times V$ of a combinatorial isolating neighborhood $\mathcal{N}$ under $\mathcal{F}$ is an isolating neighborhood for any continuous selector of $\mathcal{F}$. This definition is stronger than what is actually required to guarantee isolation on the topological level. It is, however, the definition that we will use in this work and is computable using the following algorithm.

Let $\mathcal{S} \subset \mathcal{Z}$. Set $\mathcal{N}=\mathcal{S}$, and let $o(\mathcal{N})$ be the combinatorial neighborhood of $\mathcal{N}$ in $\mathcal{Z}$. If $\operatorname{Inv}(o(\mathcal{N}), \mathcal{F})=\mathcal{N}$, then $\mathcal{N}$ is isolated under $\mathcal{F}$. If not, set $\mathcal{N}:=\operatorname{Inv}(o(\mathcal{N}), \mathcal{F})$ and repeat the above procedure. In this way, we grow the set $\mathcal{N}$ until either the isolation condition is met, or the set grows to intersect the boundary of $\mathcal{Z}$, in which case the algorithm fails to locate an isolating neighborhood in $\mathcal{Z}$. However, if the set on which the algorithm is applied is an attractor, then a neighborhood that intersects the boundary is permissible. This procedure for growing a combinatorial isolating neighborhood is outlined in more detail in [6] and [5].

Once we have an isolating neighborhood for $f$, our next goal is to compute a corresponding index pair. The following definition of a combinatorial index pair emphasizes use of an outer approximation to compute structures for $f$.

Definition 3.4 (Robbin and Salamon [17]). Let $P=\left(P_{1}, P_{0}\right)$ be a pair of compact sets with $P_{0} \subset P_{1} \subset X . P$ is an index pair provided that $\operatorname{cl}\left(P_{1} \backslash P_{0}\right)$ is an isolating neighborhood and the induced map, $f_{P}:\left(P_{1} / P_{0},\left[P_{0}\right]\right) \rightarrow\left(P_{1} / P_{0},\left[P_{0}\right]\right)$,

$$
f_{P}(x):= \begin{cases}f(x) & \text { if } x, f(x) \in P_{1} \backslash P_{0} \\ {\left[P_{0}\right]} & \text { otherwise }\end{cases}
$$

is continuous. Finally, a pair $\mathcal{P}=\left(\mathcal{P}_{1}, \mathcal{P}_{0}\right)$ of cubical sets is a combinatorial index pair for an outer approximation $\mathcal{F}$ if the corresponding topological realization $P=$ $\left(P_{1}, P_{0}\right)$, where $P_{i}:=\left|\mathcal{P}_{i}\right|$, is an index pair for any continuous selector $f$ of $\mathcal{F}$.

An algorithm is given in [5] that can be used to compute a combinatorial index pair corresponding to a combinatorial isolating neighborhood. In essence, the algorithm 
identifies the portions of the boundary of the combinatorial isolating neighborhood that act as the exit set, meaning that trajectories that leave the neighborhood must (topologically) pass through this set. The second element of the pair, $\mathcal{P}_{0}$, records the exit set.

Given an outer approximation, we now have algorithms to compute isolating neighborhoods $|\mathcal{N}|$ and corresponding index pairs $P:=\left(\left|\mathcal{P}_{1}\right|,\left|\mathcal{P}_{0}\right|\right)$ for $f$, where $|\mathcal{N}|=$ $\left|\mathcal{P}_{1} \backslash \mathcal{P}_{0}\right|$. What remains is the computation of the Conley index for the associated isolated invariant set, $S:=\operatorname{Inv}(|\mathcal{N}|, f)$.

Definition 3.5. Let $P=\left(P_{1}, P_{0}\right)$ be an index pair for the isolated invariant set $S=\operatorname{Inv}\left(\operatorname{cl}\left(P_{1} \backslash P_{0}\right), f\right)$, and let $f_{P_{*}}: H_{*}\left(P_{1}, P_{0}\right) \rightarrow H_{*}\left(P_{1}, P_{0}\right)$ be the maps induced on the relative homology groups $H_{*}\left(P_{1}, P_{0}\right)$ from the map $f_{P}$. The Conley index of $S$ is the shift equivalence class of $f_{P *}$

$$
\operatorname{Con}(S, f):=\left[f_{P *}\right]_{s} .
$$

For a definition of shift equivalence, see [9].

The Conley index for the isolated invariant set $S$ given in Definition 3.5 is well defined; namely, every isolated invariant set has an index pair, and the corresponding shift equivalence class remains invariant under different choices for this index pair; see, e.g., [14].

What remains in the computation of the index is to compute the maps $f_{P *}$ : $H_{*}\left(\left|\mathcal{P}_{1}\right|,\left|\mathcal{P}_{0}\right|\right) \rightarrow H_{*}\left(\left|\mathcal{P}_{1}\right|,\left|\mathcal{P}_{0}\right|\right)$. If the multivalued map $\mathcal{F}$ is acyclic on $\mathcal{P}_{1}$, that is, images of individual cubes in $\mathcal{P}_{1}$ have the topology of a point, then once again the combinatorial multivalued map provides the appropriate computational framework for computing these induced maps on homology as described in [10], and we use the software program homcubes in [15] to check acyclicity and compute $f_{P *}$. This step is also outlined in [5].

So far we have passed from continuous maps to induced maps on relative homology. Our overall goal, however, is to describe the dynamics of our original map. There are a number of tools that one can use to interpret Conley indices. The most basic is Ważewski's principle.

Theorem 3.1. If $\operatorname{Con}(S, f) \neq[0]_{s}$, then $S \neq \emptyset$.

In addition, we may use the Lefschetz number to draw more detailed conclusions about the dynamics.

TheOrem 3.2. Let $P=\left(P_{1}, P_{0}\right)$ be an index pair for isolated invariant set $S$. If the Lefschetz number

$$
L(S, f):=\sum_{k}(-1)^{k} \operatorname{tr}\left(f_{P k}\right)
$$

is nonzero, then $S$ contains a fixed point.

The Lefschetz number of the isolated invariant set $S$ is well defined since the Conley index of $S$ is well defined and the trace is invariant under shift equivalence. By attaching symbols to each connected component of the isolating neighborhood and computing Conley index information for maps between labeled regions, one may use a corollary of Theorem 3.2 to study symbolic dynamics. Using techniques developed in [5] to locate and validate complicated symbolic dynamics, one may also obtain lower bounds on topological entropy, one measure of chaos. This approach is described in more detail in [5] and will be applied to the outer approximation we construct for (1.1) with Ricker nonlinearity, giving one of the sample results in section 8 .

Arai et al. [1] describe a procedure for creating a database of dynamics for families of dynamical systems. Essentially, interval ranges of parameter values (for example, 
allowing $\mu$ in (1.2) to be a small interval rather than an exact value) can be directly incorporated into the construction of the outer approximation, with all maps in the model family with parameters in the included parameter set as continuous selectors. In this approach, Conley-Morse graphs attach Conley index information to partially ordered invariant sets, or Morse sets, off of which the system exhibits gradient behavior. Locating regions in parameter space over which computed Conley-Morse graphs remain invariant enables a coarse description of the dynamics within the family. For illustration, Conley-Morse information at a single parameter value set is computed for (1.1) in section 8 .

These and other techniques can be used to extract dynamics from outer approximations. Our focus in the remainder of the paper will be on the construction of outer approximations for (1.1), in which the resolution allows these tools to uncover useful information. We conclude with sample results in section 8 that demonstrate the effectiveness of our approach.

4. Outer approximation of $\boldsymbol{\Phi}$. To obtain the combinatorial approximations described in the previous section for the infinite-dimensional map (1.1), we approximate $\Phi$ in two ways. First we approximate the nonlinearity by a polynomial, and second we consider a Galerkin projection onto a finite set of Fourier modes. Even though the results of this paper apply more generally, for ease of presentation, we will assume that the operator $G[a]$ has the form $G[a](x)=\mu c(x) g(a(x))$ as in the Kot-Schaffer-Ricker map defined in (1.2).

We begin with some further smoothness assumptions on the function $g$ and some notation. Let $\left[a_{-}, a_{+}\right]$be an a priori bounding interval for $a(x)$, that is, $a_{-} \leq a(x) \leq$ $a_{+}$for all $x \in[-\pi, \pi]$. We denote the affine homeomorphisms between $[-1,1]$ and $\left[a_{-}, a_{+}\right]$by

$$
\sigma_{a}(u)=\frac{a_{+}-a_{-}}{2}(u+1)+a_{-} \quad \text { and } \quad \sigma_{u}(a)=\frac{2}{a_{+}-a_{-}}\left(a-a_{-}\right)-1 .
$$

We assume that $\left[a_{-}, a_{+}\right]$is in the domain of $g$ and that $g \circ \sigma_{a}:[-1,1] \rightarrow \mathbb{R}$ can be continued to an analytic function in a complex neighborhood of $[-1,1]$.

Fix $L>0$. Let $t_{l}=\cos (l \pi / L)$ for $l=0, \ldots, L$ be the Chebyshev-Gauss-Lobatto points in $[-1,1]$. Then the Chebyshev interpolating polynomial of $g \circ \sigma_{a}$ at these points is denoted by $P_{L}$, and $P_{L} \circ \sigma_{u}$ interpolates $g:\left[a_{-}, a_{+}\right] \rightarrow \mathbb{R}$. The convergence of these interpolants is exponential in $L$. In particular, for each $\rho>1$ there exists $C\left(a_{-}, a_{+}, \rho\right)$ such that

$$
\max _{u \in[-1,1]}\left|g\left(\sigma_{a}(u)\right)-P_{L}(u)\right|=\max _{a \in\left[a_{-}, a_{+}\right]}\left|g(a)-P_{L}\left(\sigma_{u}(a)\right)\right| \leq C\left(a_{-}, a_{+}, \rho\right) \cdot \rho^{-L} .
$$

Indeed, in section 5 we describe how to obtain explicitly computable bounds on $C\left(a_{-}, a_{+}, \rho\right)$ as well as error bounds for the first two derivatives of $g\left(\sigma_{a}(u)\right)-P_{L}(u)$ for arbitrary analytic functions.

We now decompose the map $\Phi$ using the Chebyshev approximation to $g \circ \sigma_{a}$ and a Galerkin projection onto a finite set of modes of $a$. Consider the Fourier expansion $a(x)=\sum_{n \in \mathbb{Z}} a_{n} e^{i n x}$ where $a_{n} \in \mathbb{R}$. Fix the projection dimension $M>0$, and let $a_{F}:=\sum_{|n|<M} a_{n} e^{i n x}$ and $a_{I}:=\sum_{|n| \geq M} a_{n} e^{i n x}$. Then

$$
\begin{aligned}
\Phi[a](y) & =\frac{1}{2 \pi} \int_{-\pi}^{\pi} b(x, y) G[a](x) d x \\
& =\frac{1}{2 \pi} \int_{-\pi}^{\pi} b(x, y) \mu c(x) g(a(x)) d x
\end{aligned}
$$




$$
\begin{aligned}
= & \frac{\mu}{2 \pi} \int_{-\pi}^{\pi} b(x, y) c(x) P_{L}\left(\sigma_{u}(a(x))\right) d x \\
& +\frac{\mu}{2 \pi} \int_{-\pi}^{\pi} b(x, y) c(x)\left(g(a(x))-P_{L}\left(\sigma_{u}(a(x))\right)\right) d x \\
= & \frac{\mu}{2 \pi} \int_{-\pi}^{\pi} b(x, y) c(x) P_{L}\left(\sigma_{u}(a(x))\right) d x+E^{\mathrm{Ch}}(a, L) \\
= & \frac{\mu}{2 \pi} \int_{-\pi}^{\pi} b(x, y) c(x) P_{L}\left(\sigma_{u}\left(a_{F}(x)\right)\right) d x \\
& +\frac{\mu}{2 \pi} \int_{-\pi}^{\pi} b(x, y) c(x)\left(P_{L}\left(\sigma_{u}(a(x))\right)-P_{L}\left(\sigma_{u}\left(a_{F}(x)\right)\right)\right) d x+E^{\mathrm{Ch}}(a, L) \\
= & \Phi^{(M, L)}\left(a_{F}\right)+E^{\mathrm{G}}(a, L, M)+E^{\mathrm{Ch}}(a, L),
\end{aligned}
$$

where

$$
\begin{gathered}
\Phi^{(M, L)}\left(a_{F}\right):=\frac{\mu}{2 \pi} \int_{-\pi}^{\pi} b(x, y) c(x) P_{L}\left(\sigma_{u}\left(a_{F}(x)\right)\right) d x \\
E^{\mathrm{Ch}}(a, L):=\frac{\mu}{2 \pi} \int_{-\pi}^{\pi} b(x, y) c(x)\left(g(a(x))-P_{L}\left(\sigma_{u}(a(x))\right)\right) d x
\end{gathered}
$$

and

$$
E^{\mathrm{G}}(a, L, M):=\frac{\mu}{2 \pi} \int_{-\pi}^{\pi} b(x, y) c(x)\left(P_{L}\left(\sigma_{u}(a(x))\right)-P_{L}\left(\sigma_{u}\left(a_{F}(x)\right)\right)\right) d x
$$

are the map on the $M$-dimensional projection $a_{F}$ and the errors due to the degree- $L$ Chebyshev approximation and Galerkin projections, respectively.

In general, we denote the sequence of Fourier coefficients of a function $f$ by $\hat{f}$ and a particular coefficient by

$$
[\hat{f}]_{n}=\frac{1}{2 \pi}\left\langle f(x), e^{-i n x}\right\rangle=\frac{1}{2 \pi} \int_{-\pi}^{\pi} f(x) e^{i n x} d x .
$$

However, for the variable functions $a$ and $u$ as well as the fixed functions $b$ and $c$, we will omit the hat and square brackets so that, for example, $b(x, y)=b(x-y)=$ $\sum_{n} b_{n} e^{i n(x-y)}$.

Using the Fourier expansions for $b$ and $c$, we would like to express the Fourier coefficients of the image $\Phi[a]$ as

$$
[\widehat{\Phi[a]}]_{n}=\frac{1}{2 \pi}\left\langle\Phi[a](y), e^{-i n y}\right\rangle=\Phi_{n}^{(M, L)}\left(a_{F}\right)+E_{n}^{\mathrm{Ch}}(a, L)+E_{n}^{\mathrm{G}}(a, L, M) \quad \text { for } n \in \mathbb{Z}
$$

as defined below. First we note that the affine homeomorphisms in (4.1) induce affine transformations between the Fourier coefficients $a_{n}, u_{n}$ of functions $a:[-\pi, \pi] \rightarrow$ $\left[a_{-}, a_{+}\right]$and $u:[-\pi, \pi] \rightarrow[-1,1]$ so that

$$
\begin{aligned}
{\left[\widehat{\sigma_{a} \circ u}\right]_{n} } & = \begin{cases}\frac{a_{+}-a_{-}}{2}\left(u_{0}+1\right)+a_{-} & \text {if } n=0, \\
\frac{a_{+}-a_{-}}{2} u_{n} & \text { if } n \neq 0,\end{cases} \\
{\left[\widehat{\sigma_{u} \circ a}\right]_{n} } & = \begin{cases}\frac{2}{a_{+}-a_{-}}\left(a_{0}-a_{-}\right)-1 & \text { if } n=0, \\
\frac{2}{a_{+}-a_{-}} a_{n} & \text { if } n \neq 0 .\end{cases}
\end{aligned}
$$


Hence, denoting $\left[\widehat{\sigma_{u} \circ a}\right]_{n}$ by $u_{n}$ and the coefficients of $P_{L}$ by $p_{l}$ for $l=0, \ldots, L$, we have that

$$
\begin{aligned}
& \Phi_{n}^{(M, L)}\left(a_{F}\right) \\
& =\frac{1}{2 \pi}\left\langle\frac{\mu}{2 \pi} \int_{-\pi}^{\pi} b(x, y) c(x) P_{L}\left(\sigma_{u}\left(a_{F}(x)\right)\right) d x, e^{-i n y}\right\rangle \\
& =\frac{1}{2 \pi}\left\langle\frac{1}{2 \pi} \int_{-\pi}^{\pi} b(x, y) \mu c(x) \sum_{l=0}^{L} p_{l}\left(\sigma_{u}\left(a_{F}(x)\right)\right)^{l} d x, e^{-i n y}\right\rangle \\
& =\frac{1}{2 \pi}\left\langle\frac{\mu}{2 \pi} \int_{-\pi}^{\pi} b(x, y) \sum_{l=0}^{L} p_{l} c(x)\left(\sigma_{u}\left(a_{F}(x)\right)\right)^{l} d x, e^{-i n y}\right\rangle \\
& =\frac{1}{2 \pi}\left\langle\frac{\mu}{2 \pi} \int_{-\pi}^{\pi} \sum_{k} b_{k} e^{i k(x-y)} \sum_{l=0}^{L} p_{l}\left(\sum_{j} c_{j} e^{i j x}\right)\left(\sum_{|j|<M} u_{j} e^{i j x}\right)^{l} d x, e^{-i n y}\right\rangle \\
& =\frac{\mu}{4 \pi^{2}} \int_{-\pi}^{\pi} \int_{-\pi}^{\pi} \sum_{k} b_{k} e^{i k(x-y)} \sum_{l=0}^{L} p_{l} \sum_{n_{0},\left|n_{i}\right|<M} c_{n_{0}} u_{n_{1}} \cdots u_{n_{l}} e^{i\left(n_{0}+\cdots+n_{l}\right) x} d x e^{-i n y} d y \\
& =\frac{\mu}{4 \pi^{2}} \int_{-\pi}^{\pi} \sum_{l=0}^{L} p_{l} \sum_{k, n_{0},\left|n_{i}\right|<M} b_{k} c_{n_{0}} u_{n_{1}} \cdots u_{n_{l}} e^{i\left(k+n_{0}+\cdots+n_{l}\right) x} d x \int_{-\pi}^{\pi} e^{-i(k+n) y} d y \\
& =\frac{\mu}{2 \pi} \sum_{l=0}^{L} p_{l} \sum_{n_{0},\left|n_{i}\right|<M} \int_{-\pi}^{\pi} b_{-n} c_{n_{0}} u_{n_{1}} \cdots u_{n_{l}} e^{i\left(-n+n_{0}+\cdots+n_{l}\right) x} d x \\
& =\mu b_{-n} \sum_{l=0}^{L} p_{l} \sum_{\substack{n_{0}+\sum_{i} n_{i}=n \\
\left|n_{i}\right|<M}} c_{n_{0}} u_{n_{1}} \cdots u_{n_{l}}
\end{aligned}
$$



is the map on $a_{F}$ projected onto the $n$th mode,

$$
\left.\left.E_{n}^{\mathrm{Ch}}(a, L)=\mu b_{-n} \sum_{j+m=n} c_{j}[\widehat{g(a(x)})-P_{L}\left(\widehat{\sigma_{u}(a(x)}\right)\right)\right]_{m}
$$

is the Chebyshev approximation error projected onto the $n$th mode, and

$$
\left.\left.E_{n}^{\mathrm{G}}(a, L, M)=\mu b_{-n} \sum_{j+m=n} c_{j}\left[P_{L}\left(\widehat{\sigma_{u}(a(x)}\right)\right)-P_{L}\left(\widehat{\sigma_{u}\left(a_{F}\right.}(x)\right)\right)\right]_{m}
$$

is the projection of the Galerkin error onto the $n$th mode.

Let $\left[\hat{u}_{F}\right]_{k}:=\left[\widehat{\sigma_{u} \circ a_{F}}\right]_{k}$. In convolution notation, we have

$$
\Phi_{n}^{(M, L)}\left(a_{F}\right)=\mu b_{-n} \sum_{l=0}^{L} p_{l}\left[\hat{c} *\left(\hat{u}_{F}\right)^{l}\right]_{n},
$$




$$
E_{n}^{\mathrm{Ch}}(a, L)=\mu b_{-n}\left[\hat{c} *\left(\widehat{g \circ a}-\widehat{P_{L} \circ u}\right)\right]_{n},
$$

and

$$
E_{n}^{\mathrm{G}}(a, L, M)=\mu b_{-n}\left[\hat{c} *\left(\widehat{P_{L} \circ u}-\widehat{P_{L} \circ u_{F}}\right)\right]_{n} .
$$

4.1. Evaluation of the computational map. In practice, we evaluate $\Phi_{n}^{(M, L)}$ on boxes $\tilde{a}_{F}:=\prod_{0 \leq n<M} \tilde{a}_{n} \times \prod_{n \geq M} 0$ in Fourier space. Recall that a tilde above a variable denotes an interval. For $a_{F}$ whose Fourier coefficients are in $\tilde{a}_{F}$, we write $u_{F}=\sigma_{u}\left(a_{F}\right)$. We express $u_{F}=\bar{u}_{F}+r_{F}$, where $\left[\bar{u}_{F}\right]_{k}$ is the center of the interval $\tilde{u}_{k}$ and $\left[r_{F}\right]_{k}=\left[u_{F}\right]_{k}-\left[\bar{u}_{F}\right]_{k}$. Also, $\tilde{u}_{k}=\bar{u}_{k}+\tilde{r}_{k}$. We use this center and radius decomposition of the boxes to reduce the additional errors introduced from properties of interval arithmetic such as the wrapping effect. Then

$$
\begin{aligned}
& \Phi_{n}^{(M, L)}\left(a_{F}\right)=\frac{1}{2 \pi}\left\langle\frac{\mu}{2 \pi} \int_{-\pi}^{\pi} b(x, y) c(x) P_{L}\left(\sigma_{u}\left(a_{F}(x)\right)\right) d x, e^{-i n y}\right\rangle \\
& =\frac{1}{2 \pi}\left\langle\frac{1}{2 \pi} \int_{-\pi}^{\pi} b(x, y) \mu c(x) \sum_{l=0}^{L} p_{l}\left(\sigma_{u}\left(\hat{a}_{F}(x)\right)\right)^{l} d x, e^{-i n y}\right\rangle \\
& =\frac{1}{2 \pi}\left\langle\frac{\mu}{2 \pi} \int_{-\pi}^{\pi} b(x, y) \sum_{l=0}^{L} p_{l} c(x)\left(\sigma_{u}\left(a_{F}(x)\right)\right)^{l} d x, e^{-i n y}\right\rangle \\
& =\frac{1}{2 \pi}\left\langle\frac{\mu}{2 \pi} \int_{-\pi}^{\pi} \sum_{k} b_{k} e^{i k(x-y)} \sum_{l=0}^{L} p_{l}\left(\sum_{j} c_{j} e^{i j x}\right)\right. \\
& \left.\cdot\left(\sum_{|j|<M}\left(\bar{u}_{j}+r_{j}\right) e^{i j x}\right)^{l} d x, e^{-i n y}\right\rangle \\
& =\frac{\mu}{4 \pi^{2}} \int_{-\pi}^{\pi} \int_{-\pi}^{\pi} \sum_{k} b_{k} e^{i k(x-y)} \sum_{l=0}^{L} p_{l} \sum_{n_{0},\left|n_{i}\right|<M} c_{n_{0}}\left(\bar{u}_{n_{1}}+r_{n_{1}}\right) \\
& \cdots\left(\bar{u}_{n_{l}}+r_{n_{l}}\right) e^{i\left(n_{0}+\cdots+n_{l}\right) x} d x e^{-i n y} d y \\
& =\frac{\mu}{4 \pi^{2}} \int_{-\pi}^{\pi} \sum_{l=0}^{L} p_{l} \sum_{k, n_{0},\left|n_{i}\right|<M} b_{k} c_{n_{0}}\left(\bar{u}_{n_{1}}+r_{n_{1}}\right) \\
& \cdots\left(\bar{u}_{n_{l}}+r_{n_{l}}\right) e^{i\left(k+n_{0}+\cdots+n_{l}\right) x} d x \int_{-\pi}^{\pi} e^{-i(k+n) y} d y \\
& =\frac{\mu}{2 \pi} \sum_{l=0}^{L} p_{l} \sum_{n_{0},\left|n_{i}\right|<M} \int_{-\pi}^{\pi} b_{-n} c_{n_{0}}\left(\bar{u}_{n_{1}}+r_{n_{1}}\right) \\
& \cdots\left(\bar{u}_{n_{l}}+r_{n_{l}}\right) e^{i\left(-n+n_{0}+\cdots+n_{l}\right) x} d x \\
& =\mu b_{-n} \sum_{l=0}^{L} p_{l} \sum_{\substack{n_{0}+\sum_{i} n_{i}=n \\
\left|n_{i}\right|<M}} c_{n_{0}}\left(\bar{u}_{n_{1}}+r_{n_{1}}\right) \cdots\left(\bar{u}_{n_{l}}+r_{n_{l}}\right)
\end{aligned}
$$




$$
\begin{aligned}
& =\mu b_{-n} \sum_{l=0}^{L} p_{l} \sum_{j=0}^{l}\left(\begin{array}{l}
l \\
j
\end{array}\right) \sum_{\substack{n_{0}+\sum_{i} n_{i}=n \\
\left|n_{i}\right|<M}} c_{n_{0}} \bar{u}_{n_{1}} \cdots \bar{u}_{n_{j}} r_{n_{j+1}} \cdots r_{n_{l}} \\
& =\mu b_{-n} \sum_{l=0}^{L} p_{l} \sum_{j=0}^{l}\left(\begin{array}{l}
l \\
j
\end{array}\right)\left[\hat{c} * \bar{u}_{F}^{j} *\left(r_{F}\right)^{l-j}\right]_{n} \\
& \subset \mu b_{-n} \sum_{l=0}^{L} p_{l} \sum_{j=0}^{l}\left(\begin{array}{l}
l \\
j
\end{array}\right)\left[\hat{c} * \bar{u}_{F}^{j} *\left(\tilde{r}_{F}\right)^{l-j}\right]_{n} .
\end{aligned}
$$

The last inclusion implies

$$
\Phi_{n}^{(M, L)}\left(\tilde{a}_{F}\right) \subset \mu b_{-n} \sum_{l=0}^{L} p_{l} \sum_{j=0}^{l}\left(\begin{array}{l}
l \\
j
\end{array}\right)\left[\hat{c} * \bar{u}_{F}^{j} *\left(\tilde{r}_{F}\right)^{l-j}\right]_{n},
$$

which provides an interval bound for the projection onto each Fourier mode of the computational map $\Phi^{(M, L)}$ evaluated on functions whose Fourier coefficients lie in a box $\tilde{a}_{F}$. In sections 5 and 6 we compute corresponding interval bounds on the projections of the Chebyshev and Galerkin errors, respectively. From these interval bounds we compute a combinatorial multivalued map on boxes in Fourier space that is an outer approximation of the infinite-dimensional map $\Phi$; see section 7 .

4.2. Computing the Chebyshev coefficients $q_{l}, p_{l}$. Before proceeding to the error estimates, we briefly describe the computation of the Chebyshev coefficients $p_{l}$. As in section 4 , let $q_{l}$ and $p_{l}$ be the coefficients of $P_{L}$ in the Chebyshev basis and the standard basis, respectively, so that $P_{L}(u)=\sum_{l=0}^{L} q_{l} T_{l}(u)=\sum_{l=0}^{L} p_{l} u^{l}$. The Chebyshev-Gauss-Lobatto points $t_{l}=\cos (\pi l / L)$ for $l=0, \ldots, L$ are in reverse order in $[-1,1]$ so that $t_{0}=1$ and $t_{L}=-1$. For a fixed $L$, the coefficients $q_{l}$ can be found very efficiently using the discrete fast Fourier transform (DFT) as follows. Evaluate $v_{l}=g\left(\sigma_{a}\left(t_{l}\right)\right)$. Extend the length of the vector $v$ by $v_{2 L-l}=v_{l}$ for $l=1,2, \ldots, L-1$. Use the DFT to compute the Fourier coefficients $[\hat{v}]_{k}=\frac{\pi}{L} \sum_{l=1}^{2 L} v_{l} e^{-i \pi k l / L}$ for $k=$ $-L+1, \ldots, L$. The real parts of the coefficients $[\hat{v}]_{k}$ for $k=0, \ldots, L$ are the coefficients $q_{l}$. The coefficients $p_{l}$ can be computed easily from the coefficients $q_{l}$ using the recurrence relation for the Chebyshev polynomials $T_{l}$; see $[18,3]$.

\section{Bounds on Chebyshev interpolation error.}

5.1. Exponential convergence of interpolants of analytic functions. Let $h:[-1,1] \rightarrow \mathbb{R}$ be a function which can be extended analytically to the interior of an ellipse $\mathrm{E}_{\rho}$ with foci at \pm 1 and sum of its semimajor and semiminor axes $\rho>1$, i.e.,

$$
\mathrm{E}_{\rho}=\left\{z \in \mathbb{C} \mid z=\frac{1}{2}\left(\rho e^{i \theta}+\rho^{-1} e^{-i \theta}\right) \text { for some } 0 \leq \theta \leq 2 \pi\right\} .
$$

Recall that for fixed $L>0$ the interpolating polynomial of $h$ at the ChebyshevGauss-Lobatto points $t_{j}=\cos (j \pi / N)$ for $j=0, \ldots, N$ in $[-1,1]$ is denoted by $P_{L}$. From [4],

$$
h(u)-P_{L}(u)=\frac{1}{2 \pi i} \int_{E_{\rho}} \frac{\omega_{L+1}(u)}{\omega_{L+1}(z)} \cdot \frac{h(z)}{z-u} d z,
$$

where $\omega_{L+1}(z)=T_{L+1}(z)-T_{L-1}(z)$ and $T_{n}$ is the $n$th Chebyshev polynomial. Hence $\left|\omega_{L+1}(u)\right| \leq 2$ for all $u \in[-1,1]$. 
Following arguments similar to those in [16], we estimate

$$
\begin{aligned}
\max _{u \in[-1,1]}\left|h(u)-P_{L}(u)\right| & \leq \frac{1}{2 \pi} \int_{\mathrm{E}_{\rho}} \frac{2|h(z)|}{\left|\omega_{L+1}(z)\right||z-u|}|d z| \\
& \leq \frac{\max _{z \in \mathrm{E}_{\rho}}|h(z)|}{\pi \min _{z \in \mathrm{E}_{\rho}}\left|\omega_{L+1}(z)\right|} \int_{\mathrm{E}_{\rho}} \frac{|d z|}{|z-u|} .
\end{aligned}
$$

Let $\mathrm{C}_{\rho}=\max _{z \in \mathrm{E}_{\rho}}|h(z)|$, let $\mathrm{L}_{\rho}$ denote the length of $\mathrm{E}_{\rho}$, and let $\mathrm{D}_{\rho}$ denote the distance from $\mathrm{E}_{\rho}$ to $[-1,1]$ so that

$$
\mathrm{L}_{\rho} \leq \pi \sqrt{\rho^{2}+\rho^{-2}} \quad \text { and } \quad \mathrm{D}_{\rho}=\frac{1}{2}\left(\rho+\rho^{-1}\right)-1 .
$$

Then, as in equation (2.17) of [16], we have

$$
\int_{\mathrm{E}_{\rho}} \frac{|d z|}{|z-u|} \leq \frac{\mathrm{L}_{\rho}}{\mathrm{D}_{\rho}} \leq 2 \pi \frac{\sqrt{\rho^{4}+1}}{(\rho-1)^{2}}
$$

Finally, from equation (2.12) of [16], for $z \in \mathrm{E}_{\rho}$ we have $\left|\omega_{L+1}(z)\right| \geq 2 \sinh (\eta) \sinh (\eta L)$, where $\eta=\log (\rho)$. Hence

$$
\max _{u \in[-1,1]}\left|h(u)-P_{L}(u)\right| \leq \mathrm{C}_{\rho} \cdot \frac{\sqrt{\rho^{4}+1}}{(\rho-1)^{2}} \cdot \frac{2}{\rho-\rho^{-1}} \cdot \frac{1}{\sinh (\eta L)} .
$$

Note that for fixed $\rho>1$, the exponential convergence as $L \rightarrow \infty$ as in (4.2) follows from $1 / \sinh (\eta L) \leq 2 \operatorname{coth}(\eta L) e^{-\eta L}$ and $\operatorname{coth}(\eta L) \rightarrow 1$ as $L \rightarrow \infty$.

To obtain explicitly computable bounds, we estimate $C_{\rho}$ and then estimate

$$
R_{0}(L)=\min _{\rho>1}\left\{\mathrm{C}_{\rho} \cdot \frac{\sqrt{\rho^{4}+1}}{(\rho-1)^{2}} \cdot \frac{2}{\rho-\rho^{-1}} \cdot \frac{1}{\sinh (\eta L)}\right\}
$$

by obtaining a choice of $L>0$ such that there exists $\rho>1$ such that the bound is less than some predetermined error.

Example. To illustrate this process, consider the function we want to study in the context of the Kot-Schaffer map, $g \circ \sigma_{a}:[-1,1] \rightarrow \mathbb{R}$, where $g(z)=z e^{-z}$ and $\sigma_{a}:[-1,1] \rightarrow\left[a_{-}, a_{+}\right]$is as defined in (4.1). To bound $\mathbf{C}_{\rho}$ we estimate

$$
\begin{aligned}
\mathrm{C}_{\rho}= & \max _{\theta \in[0,2 \pi]} \mid \frac{1}{2}\left(\frac{a_{+}-a_{-}}{2}\left(\rho e^{i \theta}+\rho^{-1} e^{-i \theta}+1\right)+a_{-}\right) \\
& \cdot e^{-\frac{1}{2}\left(\left(a_{+}-a_{-}\right)\left(\rho e^{i \theta}+\rho^{-1} e^{-i \theta}+1\right) / 2+a_{-}\right)} \mid \\
\leq & \frac{1}{2}\left(\frac{a_{+}-a_{-}}{2}\left(\rho+\rho^{-1}+1\right)+\left|a_{-}\right|\right) e^{-\frac{1}{4}\left(a_{+}+a_{-}\right)} e^{\left(a_{+}-a_{-}\right)\left(\rho+\rho^{-1}\right) / 4} .
\end{aligned}
$$

For $\left[a_{-}, a_{+}\right]=[-0.025,8.27]$, the final bounds on the functions $a(x)$ in the sample computation in section 8 , and $L=14$ we obtain an error bound $R_{0}(14)$ of approximately $6.7 \times 10^{-6}$ when $\rho=7.143$. 
5.2. Explicit bounds on the Chebyshev error $\boldsymbol{E}_{n}^{\mathrm{Ch}}(\boldsymbol{a}, \boldsymbol{L})$. We can improve the bounds of the previous section by incorporating estimates on the derivatives of the nonlinearity. For ease of presentation, we assume that the nonlinearity $G[a]$ in the map (1.1) can be expressed as $G[a](x)=\mu c(x) g(a(x))$ for some function $g:\left[a_{-}, a_{+}\right] \rightarrow \mathbb{R}$ with the property that $g \circ \sigma_{a}:[-1,1] \rightarrow \mathbb{R}$ can be extended analytically to the interior of an ellipse $\mathrm{E}_{\rho}$ for some $\rho>1$.

Let $a:[-\pi, \pi] \rightarrow \mathbb{R}$ be a function which has a priori bounds $\left[a_{-}, a_{+}\right]$. We want to obtain bounds on the Fourier coefficients of $\left(g-P_{L} \circ \sigma_{u}\right) \circ a:[-\pi, \pi] \rightarrow \mathbb{R}$. Since $g \circ \sigma_{a}$ can be extended analytically to the interior of an ellipse $\mathrm{E}_{\rho}$, we have

$$
g\left(\sigma_{a}(u)\right)-P_{L}(u)=\frac{1}{2 \pi i} \int_{E_{\rho}} \frac{\omega_{L+1}(u)}{\omega_{L+1}(z)} \cdot \frac{g \circ \sigma_{a}(z)}{z-u} d z .
$$

We want to establish explicit bounds on the Chebyshev approximation error projected onto the $n$th Fourier mode. We proceed as in the previous section but also estimate the error on derivatives.

In addition to the trivial bound $\left|\omega_{L+1}(u)\right| \leq 2$ for all $u \in[-1,1]$, it can also be shown that $\left|\omega_{L+1}^{\prime}(u)\right| \leq 4 L$ and $\left|\omega_{L+1}^{\prime \prime}(u)\right| \leq\left(8 L^{3}+4 L^{2}\right) / 3$ for all $u \in[-1,1]$; cf. [16]. Then, following similar arguments as before, we estimate

$$
\begin{aligned}
\max _{u \in[-1,1]}\left|\left(g \circ \sigma_{a}\right)^{\prime}(u)-P_{L}^{\prime}(u)\right| & \leq \frac{1}{2 \pi} \int_{\mathrm{E}_{\rho}}\left[\frac{\left|\omega_{L+1}^{\prime}(u)\right|}{|z-u|}+\frac{\left|\omega_{L+1}(u)\right|}{|z-u|^{2}}\right] \frac{\left|g \circ \sigma_{a}(z)\right|}{\left|\omega_{L+1}(z)\right|}|d z| \\
& \leq \frac{\max _{z \in \mathrm{E}_{\rho}}|f(z)|}{2 \pi \min _{z \in \mathrm{E}_{\rho}}\left|\omega_{L+1}(z)\right|} \int_{\mathrm{E}_{\rho}}\left[\frac{4 L}{|z-u|}+\frac{2}{|z-u|^{2}}\right]|d z|
\end{aligned}
$$

and

$$
\begin{aligned}
& \max _{a \in[-1,1]}\left|\left(g \circ \sigma_{a}\right)^{\prime \prime}(u)-P_{L}^{\prime \prime}(u)\right| \\
& \quad \leq \frac{1}{2 \pi} \int_{\mathrm{E}_{\rho}}\left[\frac{\left|\omega_{L+1}^{\prime \prime}(u)\right|}{|z-u|}+\frac{2\left|\omega_{L+1}^{\prime}(u)\right|}{|z-u|^{2}}+\frac{2\left|\omega_{L+1}(u)\right|}{|z-u|^{3}}\right] \frac{\left|g \circ \sigma_{a}(z)\right|}{\left|\omega_{L+1}(z)\right|}|d z| \\
& \quad \leq \frac{\max _{z \in \mathrm{E}_{\rho}}\left|g \circ \sigma_{a}(z)\right|}{2 \pi \min _{z \in \mathrm{E}_{\rho}}\left|\omega_{L+1}(z)\right|} \int_{\mathrm{E}_{\rho}}\left[\frac{8 L^{3}+4 L^{2}}{3|z-u|}+\frac{8 L}{|z-u|^{2}}+\frac{4}{|z-u|^{3}}\right]|d z| .
\end{aligned}
$$

As in the previous section, from [16] for $z \in E_{\rho}$ we have $\left|\omega_{L+1}(z)\right| \geq 2 \sinh (\eta) \sinh (\eta L)$, where $\eta=\log (\rho)$, and hence

$$
\max _{u \in[-1,1]}\left|\left(g \circ \sigma_{a}\right)^{\prime}(u)-P_{L}^{\prime}(u)\right| \leq \frac{1}{2 \pi} \cdot \mathrm{C}_{\rho} \cdot \mathrm{L}_{\rho} \cdot\left[\frac{2 L}{\mathrm{D}_{\rho}}+\frac{1}{\mathrm{D}_{\rho}^{2}}\right] \cdot \frac{1}{\sinh (\eta) \sinh (\eta L)}
$$

and

$$
\begin{aligned}
& \max _{u \in[-1,1]}\left|\left(g \circ \sigma_{a}\right)^{\prime \prime}(u)-P_{L}^{\prime \prime}(u)\right| \\
& \quad \leq \frac{1}{2 \pi} \cdot \mathrm{C}_{\rho} \cdot \mathrm{L}_{\rho} \cdot\left[\frac{8 L^{3}+4 L^{2}}{6 \mathrm{D}_{\rho}}+\frac{4 L}{\mathrm{D}_{\rho}^{2}}+\frac{2}{\mathrm{D}_{\rho}^{3}}\right] \cdot \frac{1}{\sinh (\eta) \sinh (\eta L)},
\end{aligned}
$$

where $\mathrm{C}_{\rho}=\max _{z \in \mathrm{E}_{\rho}}\left|g \circ \sigma_{a}(z)\right|$.

Under the change of variables $\sigma_{u}$,

$$
g^{\prime}(a)-\left(P_{L} \circ \sigma_{u}\right)^{\prime}(a)=\left(\left(g \circ \sigma_{a}\right)^{\prime}(u)-P_{L}^{\prime}(u)\right) \sigma_{u}^{\prime}(a)
$$


so that

$$
\max _{a \in\left[a_{-}, a_{+}\right]}\left|g^{\prime}(a)-\left(P_{L} \circ \sigma_{u}\right)^{\prime}(a)\right| \leq \frac{a_{+}-a_{-}}{4 \pi} \cdot \mathrm{C}_{\rho} \cdot \mathrm{L}_{\rho} \cdot\left[\frac{2 L}{\mathrm{D}_{\rho}}+\frac{1}{\mathrm{D}_{\rho}^{2}}\right] \cdot \frac{1}{\sinh (\eta) \sinh (\eta L)}
$$

and

$$
\begin{aligned}
& \max _{a \in\left[a_{-}, a_{+}\right]}\left|g^{\prime \prime}(a)-\left(P_{L} \circ \sigma_{u}\right)^{\prime \prime}(a)\right| \\
& \quad \leq \frac{\left(a_{+}-a_{-}\right)^{2}}{8 \pi} \cdot \mathrm{C}_{\rho} \cdot \mathrm{L}_{\rho} \cdot\left[\frac{8 L^{3}+4 L^{2}}{6 \mathrm{D}_{\rho}}+\frac{4 L}{\mathrm{D}_{\rho}^{2}}+\frac{2}{\mathrm{D}_{\rho}^{3}}\right] \cdot \frac{1}{\sinh (\eta) \sinh (\eta L)} .
\end{aligned}
$$

Now we estimate the projections onto the $n$th Fourier mode:

$$
\begin{aligned}
& {\left[\left(g-P_{L} \circ \sigma_{u}\right) \circ a\right]_{n}} \\
& \quad=\frac{1}{2 \pi} \int_{-\pi}^{\pi}\left(g-P_{L} \circ \sigma_{u}\right)(a(x)) e^{-i n x} d x \\
& \quad=\frac{1}{2 \pi i n} \int_{-\pi}^{\pi}\left(g-P_{L} \circ \sigma_{u}\right)^{\prime}(a(x)) a^{\prime}(x) e^{-i n x} d x \\
& \quad=\frac{-1}{2 \pi n^{2}} \int_{-\pi}^{\pi}\left[\left(g-P_{L} \circ \sigma_{u}\right)^{\prime \prime}(a(x))\left(a^{\prime}(x)\right)^{2}+\left(g-P_{L} \circ \sigma_{u}\right)^{\prime}(a(x)) a^{\prime \prime}(x)\right] e^{-i n x} d x .
\end{aligned}
$$

Define

$$
R_{1}(L)=\min _{\rho>1}\left\{\frac{a_{+}-a_{-}}{4 \pi} \cdot \mathrm{C}_{\rho} \cdot \mathrm{L}_{\rho} \cdot\left[\frac{2 L}{\mathrm{D}_{\rho}}+\frac{1}{\mathrm{D}_{\rho}^{2}}\right] \cdot \frac{1}{\sinh (\eta) \sinh (\eta L)}\right\}
$$

and

$$
R_{2}(L)=\min _{\rho>1}\left\{\frac{\left(a_{+}-a_{-}\right)^{2}}{8 \pi} \cdot \mathrm{C}_{\rho} \cdot \mathrm{L}_{\rho} \cdot\left[\frac{8 L^{3}+4 L^{2}}{6 \mathrm{D}_{\rho}}+\frac{4 L}{\mathrm{D}_{\rho}^{2}}+\frac{2}{\mathrm{D}_{\rho}^{3}}\right] \cdot \frac{1}{\sinh (\eta) \sinh (\eta L)}\right\},
$$

where the minimum is taken over all $\rho>1$ for which $g \circ \sigma_{a}$ can be extended analytically to the interior of the ellipse $\mathrm{E}_{\rho}$. Then

$$
\begin{aligned}
& \left|\left[\left(g-P_{L} \circ \sigma_{u}\right) \circ a\right]_{n}\right| \\
& \quad \leq \min \left\{R_{0}(L), \frac{R_{1}(L)\left\|a^{\prime}\right\|_{1}}{2 \pi n}, \frac{R_{2}(L)\left\|a^{\prime}\right\|_{2}^{2}+R_{1}(L)\left\|a^{\prime \prime}\right\|_{1}}{2 \pi n^{2}}\right\} \leq R(L, n) .
\end{aligned}
$$

The overall bound $R(L, n)$ can be obtained using the estimates in section 2 for a priori bounds on $\left\|a^{\prime}\right\|_{1},\left\|a^{\prime}\right\|_{2}^{2}$, and $\left\|a^{\prime \prime}\right\|_{1}$ and the values of the right-hand sides of (5.2), (5.3), and (5.4) for a fixed value of $\rho$. Estimating the Chebyshev approximation error projected onto the $n$th Fourier mode as defined in (4.5) using (5.5) yields

$$
E_{n}^{\mathrm{Ch}}(a, L) \subset \mu b_{-n} \sum_{j+k=n}\left|c_{j}\right| \cdot R(L, k) \cdot[-1,1] .
$$

By assumption, $c \in C^{2}([-\pi, \pi])$, in which case there exists $C \geq 0$ such that for $|n| \geq M,\left|c_{n}\right| \leq \frac{C}{|n|^{2}}$. By (5.5), there exists $R \geq 0$ such that

$$
|R(L, n)| \leq \begin{cases}R & \text { if } n=0 \\ \frac{R}{|n|^{2}} & \text { if }|n|>0 .\end{cases}
$$


The sum in (5.6) may be infinite. However, it can be estimated in several ways. One bound is the following and may be improved by splitting the sum into more cases:

$$
\begin{aligned}
& E_{n}^{\mathrm{Ch}}(a, L) \subset \mu b_{-n} \sum_{j+k=n}\left|c_{j}\right| \cdot R(L, k) \cdot[-1,1] \\
& \subset \mu b_{-n}\left(\sum_{\substack{j+k=n \\
|j|<M}}\left|c_{j}\right| \cdot R(L, k) \cdot[-1,1]+\frac{2 C}{M^{2}} \sum_{k} R(L, k) \cdot[-1,1]\right) \\
& \subset \mu b_{-n} \sum_{\substack{j+k=n \\
|j|<M}}\left|c_{j}\right| \cdot R(L, k) \cdot[-1,1]+\frac{2\left(3+\pi^{2}\right) C R \mu b_{-n}}{3 M^{2}} \cdot[-1,1] .
\end{aligned}
$$

Note that the sum in (5.7) is a finite (computable) sum, and the remainder term contains $b_{-n}$, which exhibits strong decay, making this term small for large $n$. In addition, if $c_{n}=0$ for $n \geq M$, then we may set $C=0$, giving a remainder term of 0 .

6. Bounds on Galerkin projection error. We now present bounds on the Galerkin error term given in (4.6),

$$
\left.\left.E_{n}^{\mathrm{G}}(a, L, M)=\mu b_{-n} \sum_{j+m=n} c_{j}\left[P_{L}\left(\widehat{\sigma_{u}(a(x)}\right)\right)-P_{L}\left(\widehat{\sigma_{u}\left(a_{F}\right.}(x)\right)\right)\right]_{m},
$$

where $P_{L}(u)=\sum_{l=0}^{L} p_{l} u^{l}$. Using a Taylor expansion of $P_{L}$ (pointwise),

$$
\begin{aligned}
P_{L}\left(\sigma_{u}(a)\right)-P_{L}\left(\sigma_{u}\left(a_{F}\right)\right)= & P_{L}^{\prime}\left(\sigma_{u}\left(a_{F}\right)\right)\left(\sigma_{u}(a)-\sigma_{u}\left(a_{F}\right)\right) \\
& +\cdots+\frac{P_{L}^{(L)}\left(\sigma_{u}\left(a_{F}\right)\right)}{L !}\left(\sigma_{u}(a)-\sigma_{u}\left(a_{F}\right)\right)^{L} \\
= & \sum_{j=1}^{L} \sum_{l=j}^{L}\left(\begin{array}{l}
l \\
j
\end{array}\right) p_{l}\left(\sigma_{u}\left(a_{F}\right)\right)^{l-j}\left(\sigma_{u}\left(a_{I}\right)-\sigma_{u}(0)\right)^{j} .
\end{aligned}
$$

Let $u_{F}=\sigma_{u}\left(a_{F}\right)$ and $u_{I}=\sigma_{u}\left(a_{I}\right)-\sigma_{u}(0)$, and note that the relationship between the Fourier coefficients of $u_{F}$ and $a_{F}$, as well as $u_{I}$ and $a_{I}$, are determined by (4.3). Then we have

$$
E_{n}^{\mathrm{G}}(a, L, M)=\mu b_{-n} \sum_{j=1}^{L} \sum_{l=j}^{L}\left(\begin{array}{l}
l \\
j
\end{array}\right) p_{l}\left[\hat{c} * \hat{u}_{F}^{l-j} * \hat{u}_{I}^{j}\right]_{n},
$$

where $*$ denotes discrete convolution and $\hat{z}^{j}$ denotes the discrete convolution of $\hat{z}$ with itself $j-1$ times.

Recall that a tilde above a variable denotes an interval. Then for $\hat{u}_{F} \in \tilde{u}_{F}:=$ $\Pi_{0 \leq n<M} \tilde{u}_{n}$ we have

$$
\begin{aligned}
& {\left[\hat{u}_{F}^{l-j}\right]_{k}=} \sum_{\substack{n_{1}+\cdots+n_{l}=k \\
\left|n_{i}\right|<M}} u_{n_{1}} \cdots u_{n_{l-j}} \\
& \subset \begin{cases}\sum_{n_{1}+\cdots+n_{l}=k} \tilde{u}_{n_{1}} \cdots \tilde{u}_{n_{l-j}} & \text { if }|k| \leq(l-j)(M-1), \\
{[0]} & \text { otherwise. }\end{cases}
\end{aligned}
$$


Once a global bound pair $\left(s, A_{s}\right)$ has been fixed for modes $n \geq M$, we have

$$
\left[\hat{a}_{I}\right]_{n} \subset \begin{cases}{[0]} & \text { if }|n|<M, \\ \frac{A_{s}}{|n|^{s}}[-1,1] & \text { if }|n| \geq M,\end{cases}
$$

which implies from (4.3)

$$
\left[\hat{u}_{I}\right]_{n} \subset \begin{cases}{[0]} & \text { if }|n|<M \\ \frac{2}{a_{+}-a_{-}} \cdot \frac{A_{s}}{|n|^{s}}[-1,1] & \text { if }|n| \geq M .\end{cases}
$$

Lemma 6.1. Suppose that for $|n| \geq M, a_{n} \in \frac{A_{s}}{|n|^{s}}[-1,1]$. Then for all $j \geq 2$ and for all $k \in \mathbb{Z}$

$$
\begin{aligned}
& {\left[\hat{a}_{I}^{j}\right]_{k} \subset \frac{2^{j-1} A_{s}^{j}}{M^{s}(M-1)^{(j-1)(s-1)}(s-1)^{j-1}}[-1,1],} \\
& {\left[\hat{u}_{I}^{j}\right]_{k} \subset \frac{2^{j}}{\left(a_{+}-a_{-}\right)^{j}} \cdot \frac{2^{j-1} A_{s}^{j}}{M^{s}(M-1)^{(j-1)(s-1)}(s-1)^{j-1}}[-1,1] .}
\end{aligned}
$$

Note that these bounds are independent of $k \in \mathbb{Z}$.

Proof. Fix $k \in \mathbb{Z}$. For $j=2$

$$
\begin{aligned}
{\left[\hat{a}_{I}^{2}\right]_{k} } & =\sum_{\substack{n_{1}+n_{2}=k \\
\left|n_{i}\right| \geq M}} a_{n_{1}} a_{n_{2}} \\
& \subset \sum_{\substack{n_{1}+n_{2}=k \\
\left|n_{i}\right| \geq M}} \frac{A_{s}}{\left|n_{1}\right|^{s}} \frac{A_{s}}{\left|n_{2}\right|^{s}}[-1,1] \\
& \subset \frac{2 A_{s}^{2}[-1,1]}{M^{s}} \sum_{n_{1} \geq M} \frac{1}{n_{1}^{s}} \\
& \subset \frac{2 A_{s}^{2}[-1,1]}{M^{s}} \int_{M-1}^{\infty} \frac{1}{x^{s}} d x \\
& \subset \frac{2 A_{s}^{2}[-1,1]}{M^{s}} \frac{(M-1)^{1-s}}{s-1} \\
& =\frac{2 A_{s}^{2}}{M^{s}(M-1)^{s-1}(s-1)}[-1,1] .
\end{aligned}
$$

Assume the formula holds for $\left[\hat{a}_{I}^{j}\right]_{k}$. Then

$$
\begin{aligned}
{\left[\hat{a}_{I}^{j+1}\right]_{k} } & =\sum_{\substack{n_{1}+n_{2}=k \\
\left|n_{1}\right| \geq M}} a_{n_{1}}\left[\hat{a}_{I}^{j}\right]_{n_{2}} \\
& \subset \sum_{\substack{n_{1}+n_{2}=k \\
\left|n_{1}\right| \geq M}} \frac{A_{s}}{\left|n_{1}\right|^{s}} \frac{2^{j-1} A_{s}^{j}}{M^{s}(M-1)^{(j-1)(s-1)}(s-1)^{(j-1)}}[-1,1] \\
& \subset \frac{2^{j-1} A_{s}^{j+1}[-1,1]}{M^{s}(M-1)^{(j-1)(s-1)}(s-1)^{(j-1)}} 2 \sum_{n_{1} \geq M} \frac{1}{n_{1}^{s}} \\
& \subset \frac{2^{j} A_{s}^{j+1}[-1,1]}{M^{s}(M-1)^{(j-1)(s-1)}(s-1)^{(j-1)}} \int_{M-1}^{\infty} \frac{1}{x^{s}} d x
\end{aligned}
$$




$$
\begin{aligned}
& \subset \frac{2^{j} A_{s}^{j+1}[-1,1]}{M^{s}(M-1)^{(j-1)(s-1)}(s-1)^{(j-1)}} \frac{(M-1)^{1-s}}{s-1} \\
& =\frac{2^{j} A_{s}^{j+1}[-1,1]}{M^{s}(M-1)^{(j)(s-1)}(s-1)^{(j)}}[-1,1] .
\end{aligned}
$$

The bounds on $\left[\hat{u}_{I}^{j}\right]_{k}$ follow from (4.3).

Note that using Lemma 6.1, for all $j \geq 2$ and $n \in \mathbb{Z}$

$$
\begin{aligned}
{\left[\hat{c} * \hat{u}_{F}^{l-j} * \hat{u}_{I}^{j}\right]_{n} \subset } & \frac{2^{j}}{\left(a_{+}-a_{-}\right)^{j}} \cdot \frac{2^{j-1} A_{s}^{j}}{M^{s}(M-1)^{(j-1)(s-1)}(s-1)^{j-1}} \\
& \sum_{k}\left[\hat{c} * \tilde{u}_{F}^{l-j}\right]_{k}[-1,1] .
\end{aligned}
$$

Combining (6.1) and (6.2) gives the final Galerkin error bound:

$$
\begin{aligned}
E_{n}^{\mathrm{G}}(B, L, M) \subset \mu b_{-n} \sum_{j=1}^{L} \sum_{l=j}^{L}\left(\begin{array}{l}
l \\
j
\end{array}\right) p_{l} \frac{2^{j}}{\left(a_{+}-a_{-}\right)^{j}} \\
\cdot \frac{2^{j-1} A_{s}^{j}}{M^{s}(M-1)^{(j-1)(s-1)}(s-1)^{j-1}} \sum_{k}\left[\hat{c} * \tilde{u}_{F}^{l-j}\right]_{k}[-1,1] .
\end{aligned}
$$

Note that the last sum is a finite sum because $\tilde{u}_{F}$ has only finitely many nonzero Fourier coefficients, and hence so does $\hat{c} * \tilde{u}_{F}^{l-j}$, even if $c$ has infinitely many nonzero coefficients.

Example. The following table, valid for all $k \in \mathbb{Z}$ by Lemma 6.1 , has been computed for the Kot-Schaffer-Ricker map with $\mu=30$ and projection dimension $M=14$ in MATLAB without interval arithmetic (values are approximate).

\begin{tabular}{|c|c|c|c|c|c|}
\hline$s$ & $A_{s}$ & {$\left[\hat{a}_{I}^{2}\right]_{k}$ bound } & {$\left[\hat{a}_{I}^{10}\right]_{k}$ bound } & {$\left[\hat{a}_{I}^{15}\right]_{k}$ bound } & {$\left[\hat{a}_{I}^{20}\right]_{k}$ bound } \\
\hline \hline 2 & 11.4 & $1.0 \times 10^{-1}$ & $9.1 \times 10^{0}$ & $1.5 \times 10^{2}$ & $2.5 \times 10^{3}$ \\
\hline 4 & 13.2 & $1.4 \times 10^{-6}$ & $8.9 \times 10^{-26}$ & $9.0 \times 10^{-38}$ & $9.2 \times 10^{-50}$ \\
\hline 10 & 1403.99 & $1.4 \times 10^{-16}$ & $8.0 \times 10^{-77}$ & $1.8 \times 10^{-114}$ & $3.9 \times 10^{-152}$ \\
\hline
\end{tabular}

7. Computational algorithms. In this section we outline how the bounds and ideas from the previous sections are put into specific algorithms to compute information about the global dynamics of the map $\Phi$ in (1.1). As described in section 3, conceptually, the computations involve a two-step process. First, a rigorous outer approximation of the global dynamics in the form of a multivalued map on a cubical grid is produced. All floating point computations are performed using interval arithmetic so that the resulting outer approximation rigorously incorporates all sources of error, which ensures that $\Phi$ is a continuous selector. Then, from such an outer approximation, which can be represented combinatorially by a directed graph, graphtheoretic algorithms and computational topological algorithms can be used to extract dynamical information for (1.1).

In practice, however, to avoid unnecessary computation, we utilize the graphtheoretic algorithms to adaptively direct the computation of the outer approximation. This allows us to focus the computations on specific sets of interest such as minimal attractors, recurrent sets, Morse sets, maximal invariant sets, or periodic sets up to some maximal period. Briefly, the process involves adaptive subdivision where at each step the dynamical set of interest is extracted, and the next subdivision is performed 
only on this set. A complete description in the context of the maximal invariant set can be found in [1].

Suppose the dispersal kernel $b$ and the nonlinearity $G$ are given. We fix various parameters, error tolerances, and error bounds before performing any computations of the map as follows. First, fix the parameter $\lambda>1$ which dominates the rate of decay of the Fourier coefficients of the kernel.

Next we fix tolerances for the Chebyshev error bounds. When the size of a domain box is large, the Galerkin projection leads to large truncation errors, so it does not make sense to waste computation on a close Chebyshev approximation. Therefore, we set the error tolerance for the Chebyshev approximation to be a factor $\epsilon>0$ of the domain box size.

Next we fix an initial tail truncation dimension $M$ and compute a priori global bounds on the computational modes. More specifically, using the estimates in (2.1), which depend only on the kernel and nonlinearity, for $k=0, \ldots, M$ set $\left[a_{k}^{-}, a_{k}^{+}\right]=$ $\frac{A_{s_{k}}}{k^{s_{k}}}[-1,1]$, where $s_{k}$ is chosen to minimize $\frac{A_{s}}{k^{s}}$ over $s \geq 2$. We fix a pair $\left(s_{*}, A_{s_{*}}\right)$ to be used for the tail modes by choosing $s_{*}=s_{M}$. Finally, we choose $m \leq M$. The bounds on the computational modes $m, \ldots, M-1$ will be updated, but we will not subdivide boxes in the grid in these modes.

The initial (Fourier) domain $Z$ obtained from the a priori global bounds is

$$
Z=R \times V=\prod_{n=0}^{M-1} \frac{A_{s_{n}}}{n^{s_{n}}}[-1,1] \times \prod_{n \geq M} \frac{A_{s_{*}}}{n^{s_{*}}}[-1,1] .
$$

As a preprocessing step, we first perform an adaptive subdivision algorithm over $Z$ where we test the pointwise bounds in (2.5) as follows. Fix $y_{1}, y_{2}, \ldots, y_{k} \in[-\pi, \pi]$. The modes $0, \ldots, m-1$ are subdivided successively based on the direction of the longest box radius. After each subdivision, if a box $B=\prod_{n=0}^{M-1} \tilde{a}_{n} \times \prod_{n \geq M} \frac{A_{s_{*}}}{n^{s_{*}}}[-1,1]$ satisfies (2.5),

$$
\left(\sum_{n<M} 2 \tilde{a}_{n} \cos \left(n y_{j}\right)+\frac{2 A_{s_{*}}}{\left(s_{*}-1\right)(M-1)^{s_{*}-1}}[-1,1]\right) \cap\left[A_{\min }\left(y_{j}\right), A_{\max }\left(y_{j}\right)\right]=\emptyset
$$

for some $y_{j}$, then we remove $B$ from the domain. After a certain number of subdivision steps we find the smallest rectangular domain

$$
\prod_{n=0}^{m-1}\left[a_{n}^{-}, a_{n}^{+}\right] \times \prod_{n=m}^{M-1} \frac{A_{s_{n}}}{n^{s_{n}}}[-1,1] \times \prod_{n \geq M} \frac{A_{s_{*}}}{n^{s_{*}}}[-1,1]
$$

which covers the remaining boxes. This improves the original a priori bounds for the computational modes in (7.1) and becomes the new domain $Z$.

After fixing the above parameters and performing the preprocessing step to shrink the domain $Z$, we perform the following adaptive subdivision algorithm, which constitutes the core part of the computation of the multivalued map. For notational purposes let $\mathcal{B}$ denote the collection of boxes at the current step, and a generic box $B \in \mathcal{B}$ is represented by $B=\prod_{n=0}^{M-1} \tilde{a}_{n} \times \prod_{n \geq M} \frac{A_{s_{*}}}{n^{s_{*}}}[-1,1]$.

1. Select the mode in $0, \ldots, m-1$ with the largest box radius, bisect each box in $\mathcal{B}$ in that direction, and update $\mathcal{B}$. This may not be the optimal choice, but without more specific information about the dynamics of the system, all choices of subdivision direction are somewhat arbitrary. One could develop adaptive strategies, but we make this choice for simplicity. 
2. For each box $B \in \mathcal{B}$ test the pointwise bounds in (2.5), and remove $B$ if the condition fails.

3. From the Chebyshev error estimate in (5.7), for each box $B \in \mathcal{B}$ choose $L$ just large enough so that for all $n=0, \ldots, M-1$ we have

$$
\begin{aligned}
E_{n}^{\mathrm{Ch}}(B, L) \subset \mu b_{-n} \sum_{\substack{j+k=n \\
|j|<M}}\left|c_{j}\right| \cdot R(L, k) \cdot[-1,1] \\
+\frac{2\left(3+\pi^{2}\right) C R \mu b_{-n}}{3 M^{2}} \cdot[-1,1] \subset \frac{1}{2} \epsilon \ell\left(\tilde{a}_{n}\right)[-1,1] .
\end{aligned}
$$

4. For each box $B \in \mathcal{B}$ let $\tilde{u}_{F}=\prod_{n=0}^{M-1} \sigma_{u}\left(\tilde{a}_{n}\right)$ and $\tilde{u}_{I}=\prod_{n=M}^{\infty} \sigma_{u}\left(\tilde{a}_{n}\right)$, and compute the Galerkin error estimates in (6.3):

$$
\begin{aligned}
E_{n}^{\mathrm{G}}(B, L, M) \subset \mu b_{-n} \sum_{j=1}^{L} \sum_{l=j}^{L}\left(\begin{array}{l}
l \\
j
\end{array}\right) p_{l} \frac{2^{j}}{\left(a_{+}-a_{-}\right)^{j}} \\
\cdot \frac{2^{j-1} A_{s}^{j}}{M^{s}(M-1)^{(j-1)(s-1)}(s-1)^{j-1}} \sum_{k}\left[\hat{c} * \tilde{u}_{F}^{l-j}\right]_{k}[-1,1] .
\end{aligned}
$$

5. For each box $B \in \mathcal{B}$ compute a bound on the finite-dimensional map $\Phi_{n}^{(M, L)}$ $\left(\tilde{a}_{F}\right)$ on the computational modes $n=0, \ldots, M-1$ using the explicit formula in (4.10),

$$
\Phi_{n}^{(M, L)}\left(\tilde{a}_{F}\right) \subset \mu b_{-n} \sum_{l=0}^{L} p_{l} \sum_{j=0}^{l}\left(\begin{array}{l}
l \\
j
\end{array}\right)\left[\hat{c} * \bar{u}_{F}^{j} *\left(\tilde{r}_{F}^{u}\right)^{l-j}\right]_{n},
$$

and add the Chebyshev and Galerkin error bounds to this bound. Cover the resulting rectangular region by boxes in $\mathcal{B}$ to produce the outer approximation $\mathcal{F}: \mathcal{B} \rightrightarrows \mathcal{B}$.

6. Using standard graph algorithms, extract the dynamical set of interest $\mathcal{S}$ from $\mathcal{B}$. Depending on the dynamics of interest, one could choose (in increasing order) the minimal attractors, the recurrent set, or the maximal invariant set. One could also extract the periodic boxes up to some maximal period. All of these sets are robust under this subdivision process. That is, the corresponding sets in the true dynamics are contained in those computed from any outer approximation $[1,11]$, and hence no dynamics is lost by restricting to $\mathcal{S}$ before further refinement.

7. For each mode $m \leq n<M$, or $0 \leq n<m$ if no subdivision has occurred in the $n$th mode, recompute the bounding interval for this fixed mode as the minimal interval $\left[a_{n}^{-}, a_{n}^{+}\right] \subset A_{s_{n}} / n^{s_{n}}[-1,1]$ which contains the image of the $n$th mode for each box in $\mathcal{S}$. If the size of these intervals is smaller than some tolerance for $M^{\prime} \leq n<M$, then redefine $M=M^{\prime}$. This change allows more modes to be covered by the tail estimates, which reduces the number of computational modes and improves the efficiency of the computations. Since we begin with an a priori global bound on the dynamics and at each stage maintain an outer approximation of the dynamics of interest, we do not need to perform any checks on the images of the modes $n \geq M$.

8. Set $\mathcal{B}=\mathcal{S}$, and repeat this process to the desired resolution. 
We make a few technical adjustments to this algorithm in practice. In particular, we set an absolute minimum error tolerance and an absolute maximum error tolerance for the Chebyshev error in step 3. For computational efficiency we do not perform both the pointwise bound test (step 2) and the multivalued map computations (steps 3 and 4) at each stage, but alternate in practice. Also, for each computation of the multivalued map we do not always recompute the Chebyshev approximation (step 3). Rather, each time the Chebyshev computations are performed, we save a universal choice of $L$ and a priori bounds $\left[a_{-}, a_{+}\right]$that work for the entire region and then use these in the next computation of the multivalued map (step 4).

Finally, we reiterate that the domain $Z=R \times V$ in (7.1) is constructed so that the regularity bounds of section 2 imply that the map $\Phi$ is forward invariant in the tail $V$. Since the computations for an outer approximation do not change the tail modes, if we construct an isolating neighborhood $N$ in $R$, then $N \times V$ is an isolating neighborhood for $\Phi$; see section 3 . In particular, the realization of the set $\mathcal{S}$ that is finally reached in step 8 above is such a neighborhood. In the next section, we further extract more specific isolating neighborhoods based on the ideas briefly described in section 3 in the context of the Kot-Schaffer-Ricker model.

8. Computations on the Kot-Schaffer-Ricker map. Following the algorithm described in section 7, we compute an outer approximation for the Kot-SchafferRicker map with $\mu=30$, and $b$ is chosen to be the function whose Fourier coefficients are $\hat{b}=\left[1,\left(\frac{1}{2}-\sum_{n=2}^{\infty} \lambda^{n}\right), \lambda^{2}, \lambda^{3}, \ldots\right]$ with $\lambda=1 / 15$. Note that these Fourier coefficients are chosen so that $b$ has average value 1 and $b_{1}$ has the largest value with $b \geq 0$, since this is a dispersal kernel for a population model. Also, $c$ is chosen to be the function whose Fourier coefficients are $\hat{c}=[0.5,0.25,0,0, \ldots]$; see Figure 8.1. In this section we present some sample results that can be derived from the outer approximation for the Kot-Schaffer-Ricker map using the tools briefly described in section 3. For related $\mathrm{C}+$ code, see [8].

Following the outline of the previous section, we fix the initial tail truncation dimension to be $M=30$ and the subdivision dimension to be $m=6$. The tail bound is computed with $s_{*}=10$. The error tolerance is set at $\epsilon=0.5$, and we impose an absolute minimum error tolerance of $10^{-5}$ and an absolute maximum error tolerance of $10^{-4}$ for the Chebyshev error. Bounds on the initial Fourier domain $Z$ in the first two modes decrease by factors of 6.4 and 5.3, respectively, after 12 subdivisions and applications of the pointwise bound test (modes $n \geq 2$ are unchanged).

We performed 33 subdivisions of this rectangular domain. At each stage we alternated between testing the pointwise bounds and computing the multivalued map


FIG. 8.1. The top curve (red) in the first plot is $\mu c(x)$, and the bottom curve (blue) is $b(x)$. The middle plot shows a sample orbit of $\Phi$. The last plot shows a sample function from each region $A, B, C, D$ of the index pair shown in Figure 8.2. 




Fig. 8.2. Projection of an index pair onto the first two modes: The magenta border is a bound on the (global) maximal invariant set (from a coarse resolution), the green boxes constitute the recurrent set of the outer approximation, and the blue and red boxes constitute an index pair for which the red boxes form an exit set. The sets $M_{1}, M_{2}, M_{3}$ are isolating neighborhoods of invariant sets that form a Morse decomposition of the maximal invariant set with the ordering $3>2>1$.

and extracting the recurrent set. The final tail truncation dimension is $M=14$, and the degree of the Chebyshev approximation at the final stage is $L=14$. The final maximal Chebyshev and Galerkin errors are of orders of magnitude $10^{-5}$ and $10^{-7}$, respectively. The size of the final recurrent set is 134,311 boxes. The results are shown in Figure 8.2. The maximal invariant set shown in the figure was computed at a coarser resolution.

This final recurrent set has three recurrent components (strongly connected path components). From $[11,1]$, these recurrent components are isolating neighborhoods for a Morse decomposition of the global dynamics. The partial order on the Morse sets is a total order, that is, $M_{3}>M_{2}>M_{1}$, where the sets $M_{i}$ are those shown in Figure 8.2. The set $M_{3}$ contains the origin and has the Conley index of an unstable source, and $M_{2}$ contains a second fixed point and has the Conley index of a saddle. The minimal Morse set $M_{3}$ is an attractor and has the Conley index of a stable period-2 cycle. However, $M_{3}$ contains more than just a period-2 orbit; indeed, we can show that there are nontrivial chaotic dynamics as follows.

First, we use graph algorithms to find all the boxes in $M_{3}$ that lie on cycles of length at most 12 . Using the algorithms briefly described in section 3 , one can rigorously show that periodic orbits of various periods exist by building appropriate index pairs around candidate cycles. In $M_{3}$ we can rigorously verify nine distinct 



FIG. 8.3. Amalgamated index pair and transition graph with lower entropy bound of 0.2406 . For sample functions in each region $A, B, C, D$ see Figure 8.1.

periodic orbits in the sense that there exist index pairs for each orbit which are mutually disjoint - one period- 2 orbit, one period- 4 orbit, two period- 6 orbits, one period- 8 orbit, two period-10 orbits, and two period-12 orbits. Finally, we can build an index pair around the entire collection of these nine cycles and construct a semiconjugacy to symbolic dynamics. The resulting index pair has 16 connected components which can be amalgamated into four regions labeled $A, B, C, D$ in Figure 8.3. The transition graph on these regions is also shown in Figure 8.3, which yields a rigorous lower bound on the topological entropy of 0.2406 . It should be emphasized that simply verifying the transition graph on these regions is not enough to establish a rigorous lower bound on entropy; information extracted from computations on the Conley index of this index pair is required to show that the entropy of the transition graph is a lower bound on the entropy of the map; see [5].

\section{REFERENCES}

[1] Z. Arai, W. Kalies, H. Kokubu, K. Mischaikow, H. Oka, and P. Pilarczyk, A database schema for the analysis of global dynamics of multiparameter systems, SIAM J. Appl. Dyn. Syst., 8 (2009), pp. 757-789.

[2] H. Ban and W. Kalies, A computational approach to Conley's decomposition theorem, J. Comput. Nonlinear Dyn., 1 (2006), pp. 312-319.

[3] J. P. Boyd, Chebyshev and Fourier Spectral Methods, 2nd ed., Dover Publications, Mineola, NY, 2001.

[4] P. J. Davis, Interpolation and Approximation, Dover, New York, 1975.

[5] S. Day, R. Frongillo, And R. Treviño, Algorithms for rigorous entropy bounds and symbolic dynamics, SIAM J. Appl. Dyn. Syst., 7 (2008), pp. 1477-1506.

[6] S. DAY, O. Junge, AND K. Mischaikow, A rigorous numerical method for the global analysis of infinite-dimensional discrete dynamical systems, SIAM J. Appl. Dyn. Syst., 3 (2004), pp. 117-160.

[7] S. Day, O. Junge, And K. Mischaikow, Towards automated chaos verification, in EQUADIFF 2003, World Scientific, Hackensack, NJ, 2005, pp. 157-162. 
[8] S. Day And W. Kalies, C++ Code for Sample Results, available online from http://math.fau. edu/kalies/KSR/software.html/, 2013.

[9] J. Franks And D. Richeson, Shift equivalence and the Conley index, Trans. Amer. Math. Soc., 352 (2000), pp. 3305-3322.

[10] T. Kaczynski, K. Mischaikow, and M. Mrozek, Computational Homology, Appl. Math. Sci. 157, Springer-Verlag, New York, 2004.

[11] W. D. Kalies, K. Mischaikow, and R. C. A. M. VanderVorst, An algorithmic approach to chain recurrence, Found. Comput. Math., 5 (2005), pp. 409-449.

[12] M. Kot And W. M. Schaffer, Discrete-time growth-dispersal models, Math. Biosci., 80 (1986), pp. 109-136.

[13] K. Mischaikow, Topological techniques for efficient rigorous computation in dynamics, Acta Numer., 11 (2002), pp. 435-477.

[14] K. Mischaikow and M. Mrozek, Conley index, in Handbook of Dynamical Systems, Vol. 2, North-Holland, Amsterdam, 2002, pp. 393-460.

[15] P. Pilarczyk, Homology Computation Software, Jagiellonian University, available online through the Computational Homology Project (Chomp) at http://chomp.rutgers. edu/software/, 1998.

[16] S. C. REDdy And J. A. C. Weideman, The accuracy of the Chebyshev differencing method for analytic functions, SIAM J. Numer. Anal., 42 (2005), pp. 2176-2187.

[17] J. W. Robbin and D. Salamon, Dynamical systems, shape theory and the Conley index, Ergodic Theory Dynam. Systems, 8* (Charles Conley Memorial Issue) (1988), pp. 375393.

[18] L. N. Trefethen, Spectral Methods in MATLAB, Software Environ. Tools 10, SIAM, Philadelphia, 2000.

[19] J. B. VAN DEN BERG AND J.-P. LESSARD, Chaotic braided solutions via rigorous numerics: Chaos in the Swift-Hohenberg equation, SIAM J. Appl. Dyn. Syst., 7 (2008), pp. 9881031. 